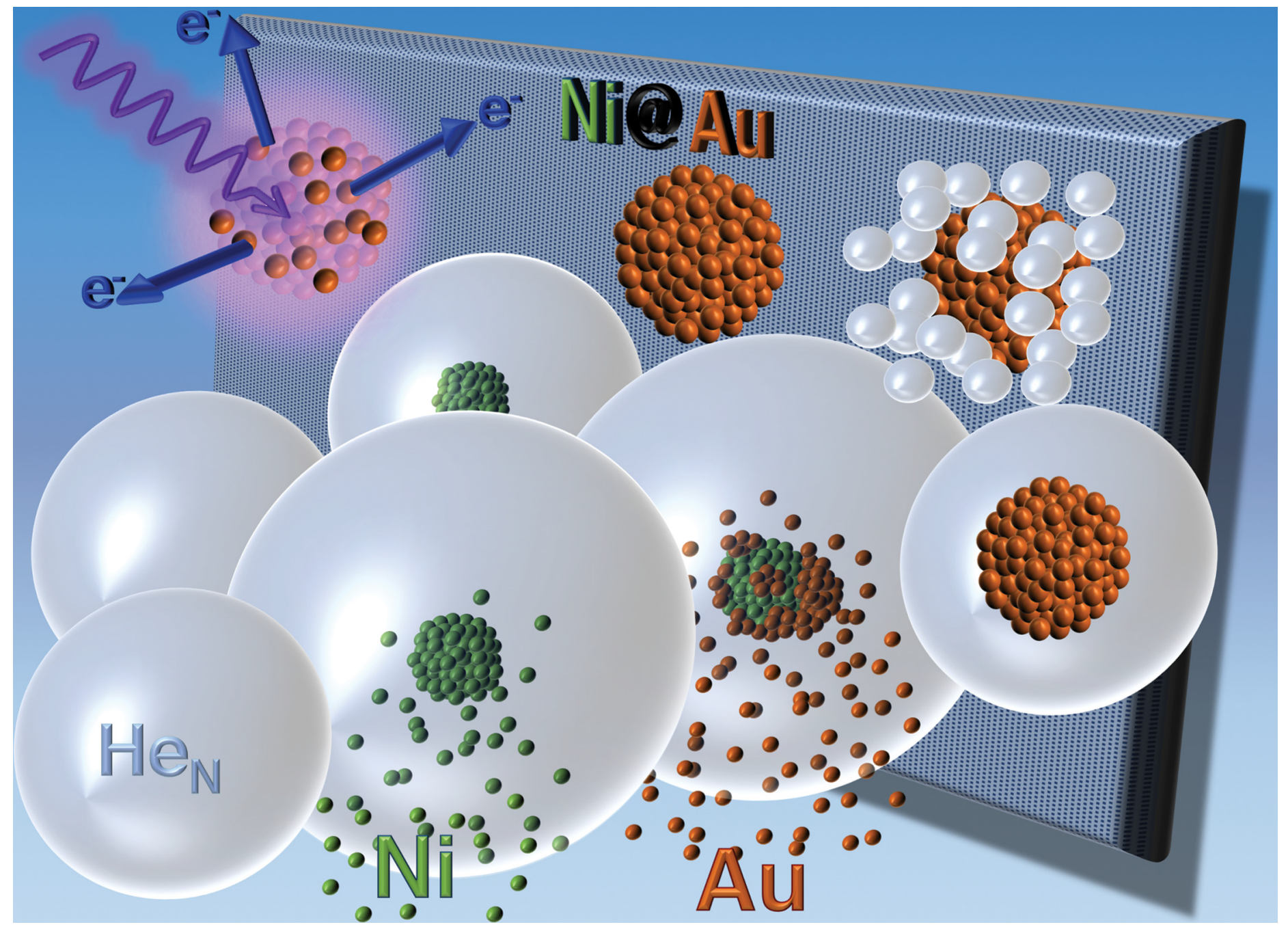

\section{Showcasing research from the Groups of}

Prof. Wolfgang E. Ernst and Prof. Andreas W. Hauser at Graz University of Technology, Graz, Austria.

Metal clusters synthesized in helium droplets: structure and dynamics from experiment and theory

We provide an overview of the high potential of using helium nanodroplets for nanoparticle synthesis. Core-shell clusters are grown by subsequent dosage of the desired atomic or molecular species into the droplets after which they are deposited and studied using diagnostics such as photoelectron spectroscopy or electron microscopy. We present the state of the art in creating mixed metal clusters in a superfluid droplet environment and the successful interplay between experimental characterization and theoretical modelling.

\section{As featured in:}

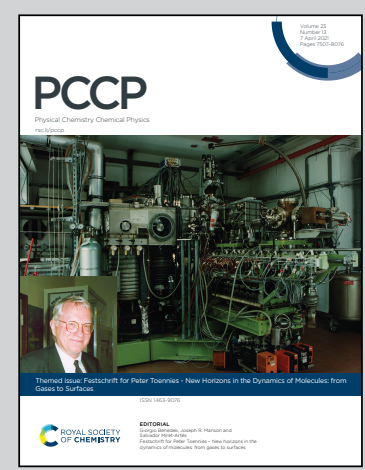

See Wolfgang E. Ernst and Andreas W. Hauser, Phys. Chem. Chem. Phys., 2021, 23, 7553. 
Check for updates

Cite this: Phys. Chem. Chem. Phys., $2021,23,7553$

Received 17th August 2020,

Accepted 29th September 2020

DOI: 10.1039/d0cp04349d

rsc.li/pccp

\title{
Metal clusters synthesized in helium droplets: structure and dynamics from experiment and theory
}

\begin{abstract}
Wolfgang E. Ernst (D)* and Andreas W. Hauser (D)*
Metal clusters have drawn continuous interest because of their high potential for the assembly of matter with special properties that may significantly differ from the corresponding bulk. Controlled combination of particular elements in one nanoparticle can increase the options for the creation of new materials for photonic, catalytic, or electronic applications. Superfluid helium droplets provide confinement and ultralow temperature, i.e. an ideal environment for the atom-by-atom aggregation of a new nanoparticle. This perspective presents a review of the current research progress on the synthesis of tailored metal and metal oxide clusters including core-shell designs, their characterization within the helium droplet beam, deposition on various solid substrates, and analysis via surface diagnostics. Special attention is given to the thermal properties of mixed metal clusters and questions about alloy formation on the nanoscale. Experimental results are accompanied by theoretical approaches employing computational chemistry, molecular dynamics simulations and He density functional theory.
\end{abstract}

Institute of Experimental Physics, Graz University of Technology, Petersgasse 16, A-8010 Graz, Austria. E-mail: wolfgang.ernst@tugraz.at,

andreas.w.hauser@gmail.com; Fax: +43-316-873-108140; Tel: +43-316-873-8157, +43-316-873-8140

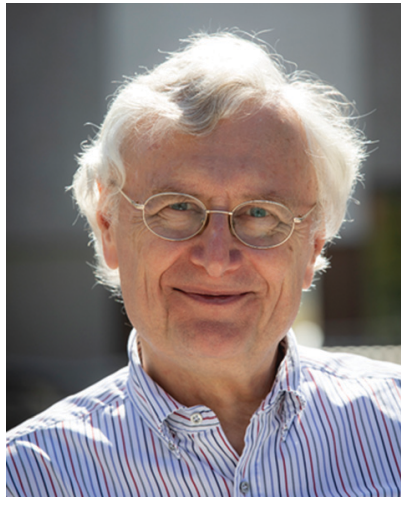

Wolfgang E. Ernst
Wolfgang E. Ernst is an Emeritus Professor of Physics at Graz University of Technology. He finished his $P h D$ in physics at TU Hannover, Germany in 1977, pursued postdoctoral research at Rice University, Houston, Texas in 1978-79, and obtained his habilitation at Freie Universität Berlin, Germany in 1983. From 1990 to 2002 he held the position of a full professor at Penn State University, USA, after which he moved to Graz University of Technology, Austria, where he served as Professor of Physics and Director of the Institute of Experimental Physics until 2019. Professor Ernst received the 1987 Physics Prize of the German Physical Society, the 1998 Penn State Faculty Scholar Medal, and in 2015 the Styrian Research Prize in Austria. He is an elected Fellow of the American Physical Society and the European Physical Society, and was President of the Austrian Physical Society from 2013 to 2014. His continuing research interests include fundamental aspects of the electronic and nuclear dynamics in molecules and at surfaces.

\section{Introduction}

Initial interest in nanoclusters or nanoparticles originated in a purely curiosity driven exploration of an intermediate state of

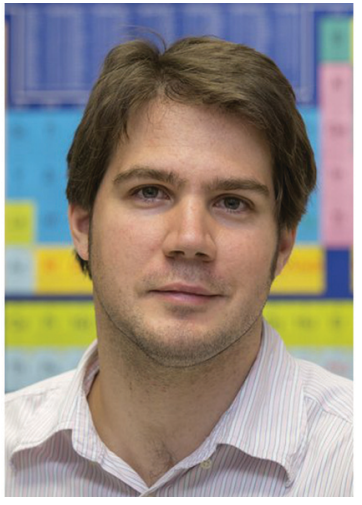

Andreas W. Hauser
Andreas Hauser is an Associate Professor at Graz University of Technology in Austria. After his years as a postdoctoral researcher at Massey University in New Zealand and UC Berkeley in California he now leads a theory group at the Institute of Experimental Physics, working in the field of materials simulations, theoretical molecular physics and electronic structure theory. Besides his work on helium droplet-related topics via helium density functional theory, embedded computational chemistry and mesoscopic simulation techniques for mixed-metallic nanoparticles, he is also interested in low-dimensional materials, heterogeneous catalysis, molecular spectroscopy and the effective application of machine learning techniques to problems of materials science. 
matter between free molecules and solid phase. Co-condensing a stream of atoms or molecules with large amounts of noble gas on a cold finger allowed an aggregation process for the production of clusters in a cold matrix. ${ }^{1}$ Early research on metal clusters was mainly based on the spectroscopy of dimers and trimers or the size-dependent measurement of ionization thresholds. $^{2}$ The two volumes on 'Clusters of Atoms and Molecules' edited by Haberland ${ }^{3}$ give an excellent overview of the field about 25 years ago. Concentrating on the electronic properties evolving with metal cluster size, de Heer summarized ionization potentials, polarizabilities, and optical properties in connection with an electronic shell model. ${ }^{4}$ Optical properties of metal clusters were the topic of a monograph by Kreibig and Vollmer around the same time. ${ }^{5}$ Applying beam deflection methods, ${ }^{6}$ de Heer and coworkers were able to assign magnetic moments via inhomogeneous magnetic fields ${ }^{7}$ or electric dipole moments via inhomogeneous electric fields ${ }^{8}$ to individual cluster sizes.

Beyond the fundamental interest, clusters as building blocks for new materials gained importance, when it was realized that 'size matters: why nanomaterials are different'. ${ }^{9}$ As a result, cluster materials became the hot topic. In order to generate a material of use, clusters have to be deposited in large quantities. On surfaces, other diagnostic methods are applicable like magnetic circular dichroism (MCD) measurements ${ }^{10,11}$ or X-ray photoelectron spectroscopy (XPS). Larger amounts of clusters can be synthesized from precursor molecules in solution by chemical methods yielding so-called ligand stabilized clusters ${ }^{12}$ with the advantage of being prevented from further coagulating and the disadvantage of changing their properties to some extent due to the presence of the ligands.

Another approach to create clusters of atoms or molecules in a cold environment is based on the development of efficient sources for generating superfluid helium droplets in beam expansions. The droplets can serve as cold traps for the accumulation of other species to form clusters that may be deposited on solid substrates. As we will show, this method has the advantage of producing nanoparticles of virtually any combination of elements in the inert environment of superfluid helium, free of any chemicals or additives. With all processes including the deposition taking place in ultrahigh vacuum, contaminations of the produced samples are excluded.

This article is structured in the following way: the next section will provide some general background on the generation of helium droplets, their function as cold matrix, and the doping with metals. Experimental techniques for the investigation of metals in droplets and after deposition will be described in a separate chapter followed by a section explaining the applied theoretical methods for modelling the aggregation process, the soft landing on a substrate, and the observed structure and dynamics of deposited metal clusters with regard to temperature dependent nanoscale phase transitions. Selected results will exemplify the advantages of this seemingly exotic method to generate metal clusters. The article will conclude with a summary and an outlook toward future developments.

\section{Helium droplets as cold matrix}

About three decades ago, helium clusters were recognized to present an excellent opportunity for the experimental study of collective phenomena in a finite size quantum system. The heliumhelium interaction is so weak that it took rather sophisticated experiments to even prove the existence of helium dimers. ${ }^{13}$ Aggregation of helium atoms to clusters requires a supersonic expansion from a cold nozzle which was realized as early as 1961. ${ }^{14}$ Later, doping rare gas clusters with foreign atoms gained interest and allowed to probe the van der Waals forces between the dopant and the surrounding rare gas, i.e. the solvation in a finite size weakly interacting medium. ${ }^{15,16}$ In connection with the topic of finite size superfluidity, the community more frequently has used the name helium nanodroplets or just helium droplets, abbreviated as $\mathrm{He}_{N}$. Spectroscopy of dopant atoms and molecules in helium droplets provided an indirect measure of the internal properties of the helium aggregate itself such as the temperature inside and at the surface, vorticity, and an idea about collective oscillations. The temperature inside helium droplets was determined as $0.37 \mathrm{~K}$ from the relative intensities of lines in rotationally resolved spectra of the $\mathrm{SF}_{6}$ molecule in helium droplets. ${ }^{17}$ Alkali atoms were observed to reside on the surface of $\mathrm{He}_{N}$ and the measurement of populations of Zeeman sublevels in the a ${ }^{3} \Sigma_{\mathrm{u}}^{+}$ lowest triplet state of $\mathrm{Rb}_{2}$ and $\mathrm{K}_{2}$ molecules yielded the same temperature, ${ }^{18}$ at the same time showing the fast relaxation of these levels to the internal helium temperature. The narrow linewidth of the OCS spectra in ${ }^{4} \mathrm{He}_{N}$ in contrast to corresponding recordings in ${ }^{3} \mathrm{He}_{N}$, were taken as clear evidence of the finite size superfluidity of ${ }^{4} \mathrm{He}_{N}$ droplets. ${ }^{19}$ Additional evidence was provided by the observation of special features in the phonon wings of spectra of molecules in ${ }^{4} \mathrm{He}_{N}$ that correspond to the roton and maxon peaks in the dispersion curve of bulk superfluid helium obtained by neutron scattering, i.e. elementary excitations in He II. $^{20}$ By femtosecond X-ray coherent diffractive imaging Vilesov, Gessner, and colleagues succeeded to observe vorticity in ${ }^{4} \mathrm{He}_{N}$ droplets, the signs of quantized collective rotations as evidence of superfluidity. ${ }^{21}$ For a long time, the spectroscopy of doped helium droplets mainly served the purpose to explore the influence of the helium surrounding on line widths and shifts, but slowly the new opportunities for the creation of new weakly bound molecular complexes were realized and applied. The probability for the survival of doped droplets was found to be larger if the coagulating species in or on droplets set less energy free. The formation of triplet ground state alkali dimers ${ }^{22}$ or quartet state trimers ${ }^{23}$ 'boils off' fewer helium atoms from the droplet than that of singlets or doublets, respectively. Nauta and Miller identified new aggregates such as 'Long Chains of Polar Molecules in Superfluid Helium, ${ }^{24}$ and 'Cyclic Water Hexamer in Liquid Helium: The Smallest Piece of Ice'. ${ }^{25}$ The dynamics of quartet state alkali trimers after laser excitation was followed ${ }^{26}$ and more homoand heterogeneous trimers were investigated in frequency ${ }^{27,28}$ and time domain. ${ }^{29}$ Larger alkali clusters from a certain size on, seem to merge into helium droplets, ${ }^{30}$ with questions about 
high-spin state stability remaining. ${ }^{31,32}$ Metal ions tend to take center positions in helium droplets with a polarized helium shell, resulting in a so-called snowball. ${ }^{33}$ Among numerous reviews during the first 10 years of spectroscopy of doped helium droplets, the article by Toennies and Vilesov ${ }^{34}$ gives an excellent overview, while a chapter in the 'Handbook of High-resolution Spectroscopy' ${ }^{35}$ provides a detailed review of spectroscopic methods covering the range from vacuum ultraviolet to microwave frequencies. The synthesis of metal clusters other than alkali or alkaline earth that were extensively studied (for alkali metals see the references above, for alkaline earth elements see e.g. ref. 36-38) requires the construction of high temperature efficient metal doping cells inside the helium droplet machines. High temperature metals can be pulselaser ablated with the helium cluster beam passing through the metal vapour cloud. With a kilohertz repetition rate laser, this metal pick-up has been combined with continuous helium cluster beams ${ }^{39}$ or even more efficiently with pulsed helium cluster beams. ${ }^{40}$ A better control of the atomic vapour pressure in the pick-up region can be achieved by thermodynamic heating or controlled continuous electron bombardment of a crucible. In our lab, a home-built electron bombardment oven for up to $1700{ }^{\circ} \mathrm{C}$ was applied to study chromium in helium droplets $^{41}$ and could be extended to max. $2000{ }^{\circ} \mathrm{C}$, while for $\mathrm{Cu},{ }^{42} \mathrm{Ag},{ }^{43} \mathrm{Au},{ }^{44} \mathrm{Pd}$, and $\mathrm{Co}, \mathrm{Ni}, \mathrm{Fe}^{45}$ or $\mathrm{In}^{46}$ sufficient vapour pressure is generated around 1000 to $1300{ }^{\circ} \mathrm{C}$ or lower and ohmically heated ovens are used. In the following, the experimental methods for the creation of metal clusters of different sizes in helium droplets, their diagnostic in the beam apparatus, deposition on different solid surfaces, and the investigation of deposited metal clusters are described.

\section{Experimental methods for cluster synthesis}

\subsection{Synthesis of metal clusters and diagnostics in the droplet beam}

Helium droplets are generated in a supersonic expansion of high pressure helium gas through a cold nozzle. Conditions for clustering of helium atoms range typically from 10 to $20 \mathrm{~K}$ nozzle temperature and 20 to 80 bar pressure before expansion. At nozzle temperatures between 6 and $10 \mathrm{~K}$, liquid helium exits the nozzle with fragmentation into large droplets. Below $4 \mathrm{~K}$, a liquid helium jet was observed. ${ }^{47}$ The Vilesov group explored the size dependence of helium droplets on the expansion parameters in detail. ${ }^{48}$ For a rough estimate, eqn (1) lists approximate droplet sizes for operation with a nozzle of $5 \mu \mathrm{m}$ diameter and 20 to 40 bar backing pressure, and the corresponding droplet sizes ( $N$ in atoms per droplet):

$$
\begin{aligned}
& T=12-20 \mathrm{~K}, \quad N=10^{4}-10^{3} \\
& T=6-10 \mathrm{~K}, \quad N=10^{8}-10^{5} \\
& T=3-5 \mathrm{~K}, \quad N \approx 10^{10}
\end{aligned}
$$

Doping a droplet with foreign atoms or molecules deposits energy into the droplet that will be dissipated by evaporation of helium atoms from the droplet. In eqn (2), the dissociation energy for $\mathrm{He}_{N} \rightarrow \mathrm{He}_{N-1}+\mathrm{He}$ is expressed in various different units that may be convenient to spectroscopists or chemists depending on their need:

$E_{\mathrm{diss}} \approx 7 \mathrm{~K} \approx 5 \mathrm{~cm}^{-1} \approx 0.6 \mathrm{meV} \approx 60 \mathrm{~J} \mathrm{~mol}^{-1} \approx 14 \mathrm{cal} \mathrm{mol}^{-1}$

When picking up metal atoms in order to form a metal cluster, it is mainly the metallic binding energy that needs to be dissipated. For example, the bulk binding energies for $\mathrm{Ag}$ and $\mathrm{Au}$ are $3 \mathrm{eV}$ and $3.8 \mathrm{eV}$ per atom, respectively, while the kinetic energy upon impact of a dopant atom onto the helium droplet is about 2 orders of magnitude smaller. Generating silver or gold clusters with $10^{5}$ to $10^{6}$ atoms, requires large helium nanodroplets with approximately $10^{10}$ helium atoms. ${ }^{49}$ The potential of helium droplets for the synthesis of clusters was realized early, see e.g. ref. 34, but it took until 2007 that Vilesov and coworkers ${ }^{50}$ provided useful formulae and clear estimates for the necessary size and flux of helium nanodroplets for assembling metal clusters of a few hundred atoms. In the years afterwards, demonstrations of the production of metal clusters boomed, notably by the groups of Vilesov, ${ }^{51}$ Ellis and Yang, ${ }^{52}$ Lindsay, ${ }^{53}$ and us. ${ }^{54}$ Fig. 1 displays our droplet beam apparatus for assembling metal clusters as it has been described also in ref. 55 .

In the source chamber, helium gas (99.9999\% purity) at a stagnation pressure of 20-60 bar is expanded through a $5 \mu \mathrm{m}$ diameter nozzle which is cooled by a closed cycle refrigeration unit (Sumitomo RDK-408D2, cooling power $1 \mathrm{~W}$ at $4 \mathrm{~K}$ ) down to only a few Kelvin. The expansion into high vacuum $\left(\approx 10^{-5} \mathrm{mbar}\right)$ results in a beam of superfluid $\mathrm{He}_{N}$ with an internal temperature of $0.37 \mathrm{~K} .^{34,35}$ The droplet sizes can be controlled by the temperature and the stagnation pressure of the helium gas. Typically, a setup with a pressure of 20 bar at $8 \mathrm{~K}$ is used for the synthesis of spherical nanoparticles, resulting in a He droplet diameter of about $70 \mathrm{~nm}$ with a mean number of about $5 \times 10^{6} \mathrm{He}$ atoms. ${ }^{48}$ For extended wire-like particles that aggregate along vortices in larger droplets, the parameters are changed to 20 bar and $5 \mathrm{~K}$, leading to $\mathrm{He}_{N}$ with diameters of around $1 \mu \mathrm{m}$, and consisting of about $2 \times 10^{10} \mathrm{He}$ atoms. ${ }^{48}$ Subsequently, the collimated $\mathrm{He}_{N}$ beam passes through another chamber $\left(\approx 10^{-7}\right.$ mbar $)$ where resistively heated tungsten baskets coated with aluminium oxide (no. 4 and 6 in Fig. 1) are used as pickup cells for the evaporation of high purity metals. Only chromium required a different oven (see above). While passing through the respective metal vapor, the $\mathrm{He}_{N}$ are doped with the evaporated atoms. A gas pickup cell (no. 5) is optional and can serve for adding reactive species like oxygen. The superfluidity of the $\mathrm{He}_{N}$ matrix enables an unobstructed and frictionless roaming of dopant atoms. Upon statistical collisions within the $\mathrm{He}_{N}$, the dopants accumulate and start to form clusters. ${ }^{56}$ Initially, these clusters are grown as multicenters inside a droplet before they further agglomerate to larger structures. The final size of the nanoparticles depends on the doping rate and on the initial $\mathrm{He}_{N}$ size, since both affect the average time between collisions and, thereby, the growth rate. ${ }^{49}$ The synthesis of wire-like nanoparticles is enabled by the presence of vortices in larger He droplets with diameters of several $100 \mathrm{~nm}$. These vortices 


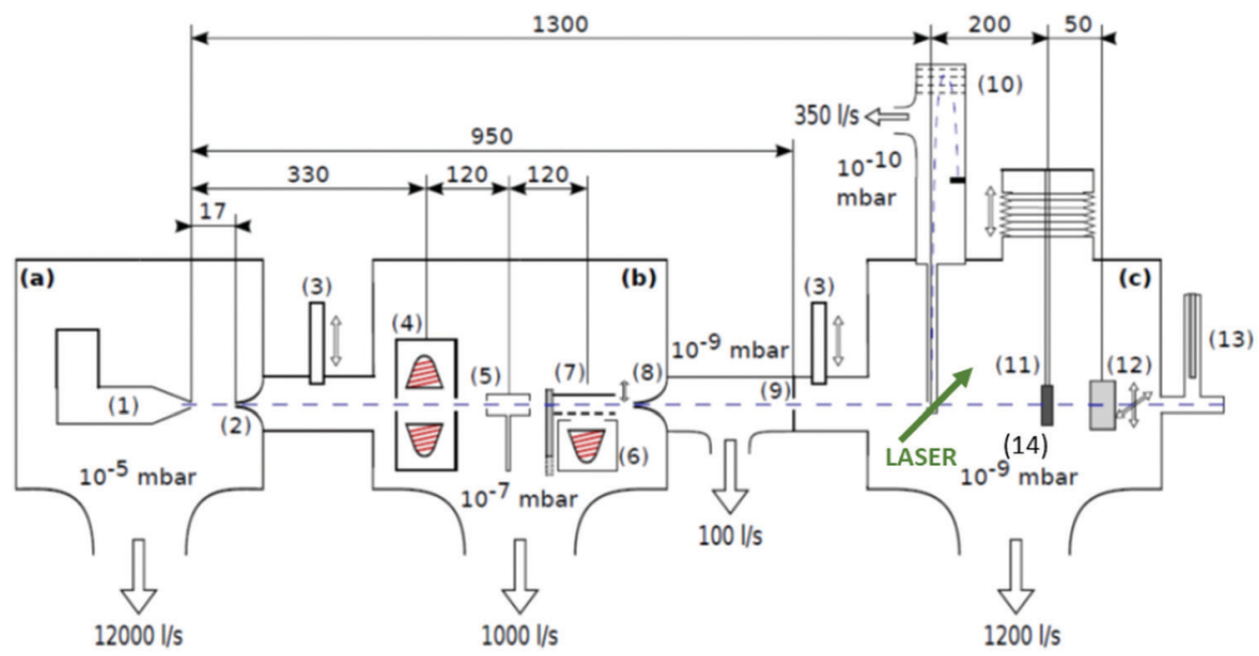

Fig. 1 Schematics of the three chamber setup ((a) SC, (b) PC, (c) DC) of the $\mathrm{He}_{N}$ beam machine used for the experiments described in this paper, see also ref. 55. The droplet beam produced by the cooled nozzle (1) is shaped by the skimmers $(2,8)$ and an aperture (9). The droplets can be sequentially doped using different sources $(4,5,6)$; (7) beam flag to engage pickup (6) (the solid line symbolises the opened state). As diagnostic tools a TOFMS (10), substrates for ex situ TEM investigations (11) and a quartz crystal microbalance (12) are available on axis, as well as an off-axis QMS (13). The TOFMS is equipped with an electron gun for electron impact ionization (not shown) but also allows photoionization using an external laser. A UHV-transfer system (14) enables the transport of surface deposited samples to external diagnostics without breaking the vacuum. Reproduced from ref. 55 [P. Thaler, A. Volk, D. Knez, F. Lackner, G. Haberfehlner, J. Steurer, M. Schnedlitz and W. E. Ernst, J. Chem. Phys., 2015, 143, 134201], with the permission of AIP Publishing.

form due to the angular momentum acquired by the superfluid droplets during the expansion of the helium through the nozzle into vacuum. ${ }^{21,57}$ The dopant metal atoms and grown clusters inside the droplets can agglomerate along such vortices due to a pressure gradient directed towards the vortex core. ${ }^{58}$ By adjusting the size of the droplets accordingly and by doping enough material into them, elongated wire-like structures can be formed along the vortices. ${ }^{59-62}$ As stated above for the two employed parameter settings in our experiments, a change in the helium source conditions directly influences the size of the $\mathrm{He}_{N}$ and allows for a facile switching between the different nanoparticle types. It should be noted that by decreasing the source temperature not only the droplet size is increased, also the total $\mathrm{He}_{N}$ flux and the deposition rate are enhanced. During agglomeration of atoms and clusters, the released binding energy is dissipated by the evaporation of He atoms from the droplet. The attenuation of the $\mathrm{He}_{N}$ beam due to the decrease in droplet size caused by this process is measured in order to monitor the particle growth process. ${ }^{49}$ The synthesis of core@ shell particles is enabled by the subsequent doping of two different metals in consecutive pickup cells. ${ }^{43,60}$ Before entering the second pickup cell holding the shell material, the particle cores have already formed with the metal provided in the first cell. The metal doped to the droplets in the second pickup cell forms a shell layer around the core. The dopant vapor pressure in each cell and, thereby, the metal atom doping rate and the nanoparticle growth are directly controlled by the temperature of the resistively heated pickup cells. Since the pickup cells are separately controlled, the amount of doped core and shell material can be adjusted independently. This allows for a tailoring of the core diameter and the shell thickness of the synthesized nanoparticles within a certain range, as has been shown for $A g @ A u^{63}$ and Fe@Au. ${ }^{64}$ After leaving the pickup region, the $\mathrm{He}_{N}$ beam reaches a third chamber at UHV conditions $\left(\approx 5 \times 10^{-10} \mathrm{mbar}\right.$ ), where a time-of-flight mass spectrometer (TOFMS) with reflectron (Stefan Kaesdorf RFT50) is mounted, which is specifically designed for the detection of large compounds. It is used for the in situ analysis of the clusters formed in the $\mathrm{He}_{N}$ and enables both electron impact ionization and laser ionization. The built-in electron gun can be tuned in energy $(0-89 \mathrm{eV})$, repetition rate $(0-10 \mathrm{kHz})$, and duty cycle $(0-20 \mu \mathrm{s})$. In most experiments presented in this work, the highest available ionization energy $(89 \mathrm{eV})$ with approximately $10 \mu \mathrm{A}$ average electron emission current at $5 \mathrm{kHz}$ repetition frequency and $20 \mu$ s duty cycle was chosen in order to get the maximum ionization cross section for He. Fig. 2 shows an example of gold clusters aggregated in $\mathrm{He}_{N}$. The higher the oven temperature, the wider the mean cluster size is shifted to the right, i.e. toward bigger clusters. The intensity drop for $T_{\mathrm{TC}}=1250{ }^{\circ} \mathrm{C}$ indicates that too many helium droplets have been extinguished by too high a load of gold atoms. A part of the mass spectrum of silver clusters created in helium droplets, is depicted in Fig. 3. The odd even oscillations are caused by the fact that an odd numbered cluster can be ionised more easily, i.e. has a lower ionisation potential as an even numbered cluster because of the unpaired electron. Magic numbers are indicated that refer to higher stability of those cluster sizes (black numbers) ascribed to closed electronic shells in the spherical jellium model. ${ }^{65}$ The high abundance of the cluster of $55 \mathrm{Ag}$ atoms may be due to the predicted structural stability of an icosahedral silver cluster. ${ }^{66}$

As had been observed by the Scheier group, ${ }^{67,68}$ ionization seems to be less destructive to aggregates if they are immersed in He nanodroplets compared to direct electron ionization of 


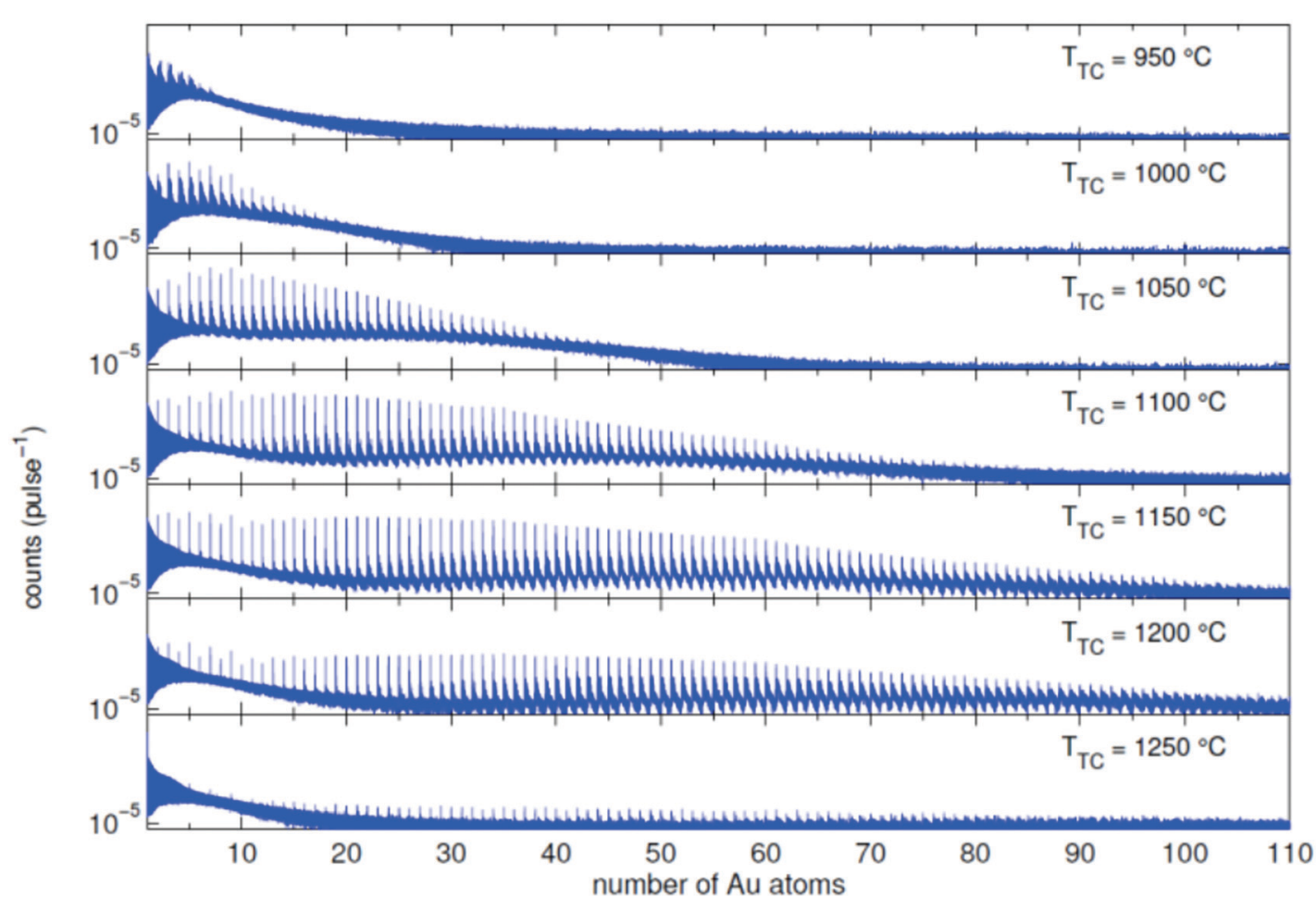

Fig. 2 Mass spectra of an Au doped He droplet beam $\left(T_{0}=9 \mathrm{~K}\right.$ and $\left.p_{0}=20 \mathrm{bar}, N_{\mathrm{He}} \approx 1.8 \times 10^{6}\right)$ for different oven temperatures $T_{\mathrm{TC}}$. Additional peaks appear due to the adsorption of water molecules in the pickup chamber. ${ }^{70}$

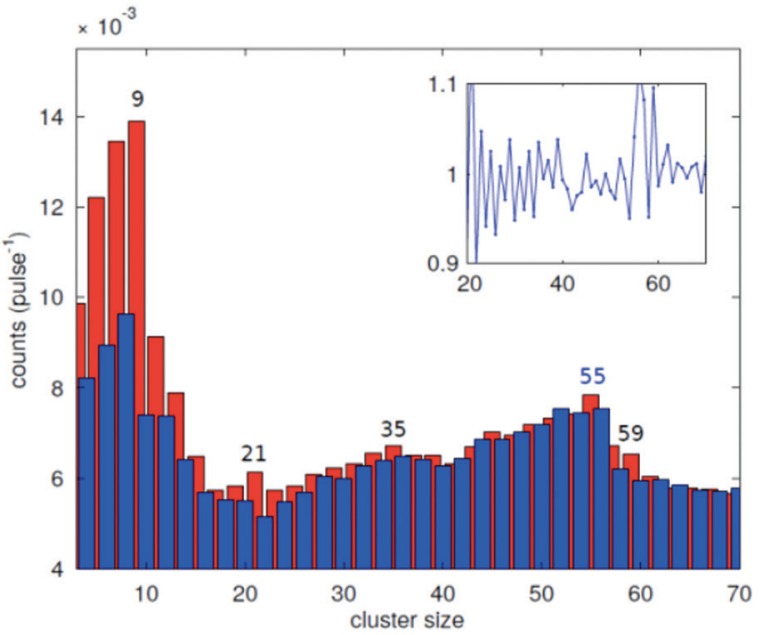

Fig. 3 Abundance of the different cluster sizes for an Ag doped $\mathrm{He}$ droplet beam. The red and blue bars mark the odd and even numbered cluster sizes, respectively. The inset shows the ratio of adjacent peaks. The magic numbers are indicated. ${ }^{70}$

the free aggregates. Probably the more indirect ionization through a charge hopping process inside the droplets and the high cooling rate of the surrounding helium are reasons for this softer process, which allowed us recently to measure a distribution of unfragmented $\mathrm{V}_{2} \mathrm{O}_{5}$ clusters. ${ }^{69}$

As latest improvement of the apparatus, another laser interaction region was added with an optical detection channel to measure integrated or wavelength selected laser induced fluorescence in a scheme previously employed in our other helium droplet experiments, see e.g. ref. 71.

Laser-optical spectroscopy of metal clusters has usually been restricted to small sizes, e.g. via setups monitoring the laserinduced fluorescence as in the mentioned case of alkali trimers, or by resonant multiphoton ionization as for $\mathrm{Au}$ oligomers. ${ }^{44}$ Tiggesbäumker, Meiwes-Broer and coworkers used two-photon laser excitation to measure the shift of the optical absorption of $\mathrm{Ag}_{8}$ in a helium droplet when exposed to additional rare gas impurities, ${ }^{72}$ which triggered a recent theoretical study on the medium-induced change of the optical response of metal clusters in rare-gas matrices. ${ }^{73}$

A quadrupole mass spectrometer (QMS) at the end of the beam path (see Fig. 1) monitors the particle flux. Set at mass number 8 that occurs upon fragmentation of helium aggregates, the intensity of the helium droplet beam can be checked. Upon each pickup of a dopant, the energy dissipation requires helium atoms to leave the droplet which diminishes the intensity of the QMS mass 8 signal. A reasonable estimate of the mean number of dopant atoms $n_{x}$ per droplet is then given by ${ }^{51}$

$$
n_{x}=N_{\text {evap }} \times E_{\mathrm{He}} / E_{X},
$$

with $E_{\mathrm{He}}=0.64 \mathrm{meV}$ being the binding energy of one helium atom to the droplet and $E_{X}$ the energy transferred to the $\mathrm{He}_{N}$ by one dopant of species $X$ (see also ref. 49).

The QMS at the end of the beam path can also be employed for beam depletion spectroscopy of larger metal clusters if the wavelength of a counterpropagating laser beam is scanned and the number of arriving metal-loaded droplets is monitored. 
Resonant excitation of a metal surface plasmon will lead to a blow up of the droplet and deplete the signal. ${ }^{74}$

\subsection{Deposition of nanoparticles and diagnostic tools}

Before the actual production of cluster samples, the deposition rate can be tested via the quartz crystal microbalance (INFICON QCM “IPN 074-156”). With temperature stabilization, we have reached a long term stability of $\pm 0.002 \mu \mathrm{g} \mathrm{cm} \mathrm{cm}^{-2} \mathrm{~s}^{-1}$ in the idle state. Using the QCM, not only the nanoparticle mass deposition rate, but also mass gain or loss due to chemical reactions can be measured. For this purpose, by controlled dosing of a reactant gas through a precision leak valve, the chemical reaction changes the mass of the deposited particles, which is detected time resolved. This way, chemical reaction rates can be determined. After such tests, newly created samples should be investigated with typical surface diagnostics not implemented in the helium droplet machine. Different substrates are necessary depending on the diagnostic tools such as electron microscopy or optical absorption spectroscopy. For deposition of the nanoparticles the substrates are inserted into the beam path with a wobblestick transfer system coupled to a vacuum suitcase (Ferrovac $\mathrm{GmbH}$, NexGeneration UHV Suitcase). The suitcase allows for the storage and transport of the samples at UHV conditions $\left(10^{-10} \mathrm{mbar}\right)$. Due to the different employed analysis methods, support grids for scanning transmission electron microscopy (STEM) (Ted Pella Inc., Ultra-thin Carbon Film on Lacey Carbon Support Film, 300 mesh, Au) as well as silicon nitride, self-prepared hexagonal boron nitride, ${ }^{75}$ or glass coverslips coated with indium tin oxide
(ITO) (spi Supplies, 30-60 $\Omega, 18 \times 18 \mathrm{~mm}$, No. 1) were used as substrates. As the $\mathrm{He}_{N}$ beam is terminated at the substrate, the particles are cushioned by the surrounding helium and, therefore, keep their shape upon deposition under soft landing conditions ${ }^{76,77}$ as observed by atomic resolution electron tomography. ${ }^{78}$ The primary analysis tool so far has been electron microscopy at the neighbouring Institute of Electron Microscopy and Nanoanalysis where various instruments are available such as several transmission electron microscopes (TEM) and an atomic resolution scanning transmission electron microscope FEI Titan3 60-300 STEM with an X-FEG field emission source operated at a beam energy of $300 \mathrm{keV}$. The STEM system is equipped with an FEI Super-X fourquadrant detector for energy dispersive x-ray spectroscopy (EDXS). Elemental mapping is provided by EDXS and electron energy loss spectroscopy (EELS). The STEM instrument is prepared to take images under a large range of tilting angles for a tomographic reconstruction of the three-dimensional shape of the investigated nano object. ${ }^{78}$ In practice, a measurement time slot at the STEM has to be efficiently used which means that metal clusters of different size may be synthesized applying different beam conditions but deposited on the same substrate. Fig. 4 depicts two Ni clusters of different size that have been exposed to air. With EELS, the oxygen coverage (blue) on the nickel (red) is obvious.

For temperature dependent studies, substrates had to be prepared that allow heating or cooling. Two TEM grids used for such studies are shown in Fig. 5. As the scale is very large, the elongated $\mathrm{Ag}$ nanoparticles appear just as little bright spots.

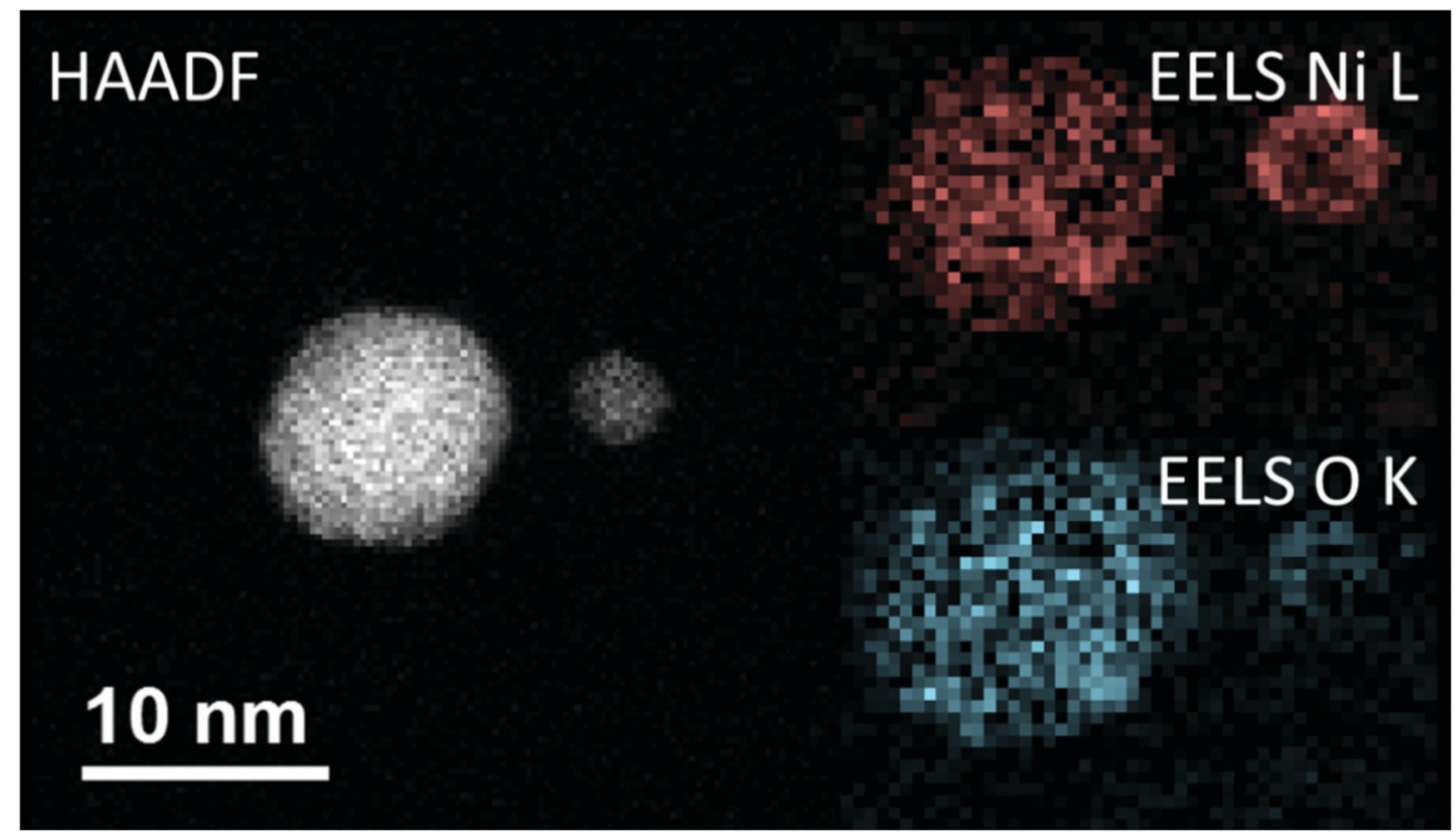

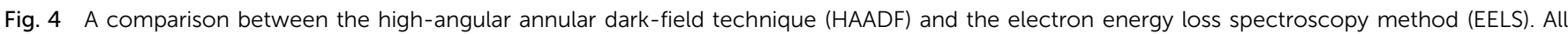

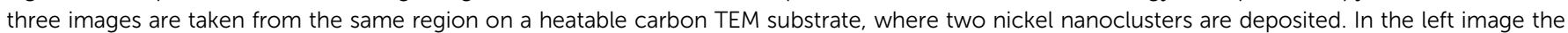

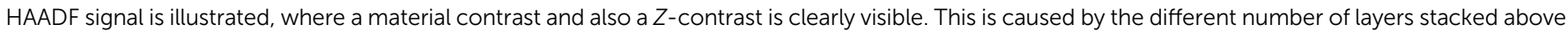

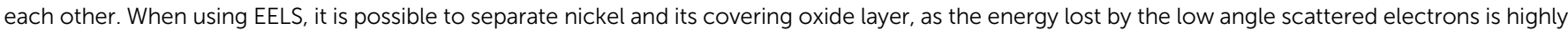

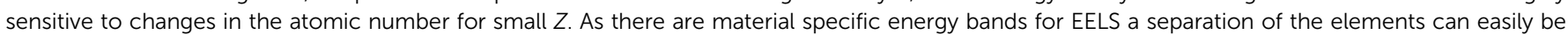
done. Taken from ref. 79 . 


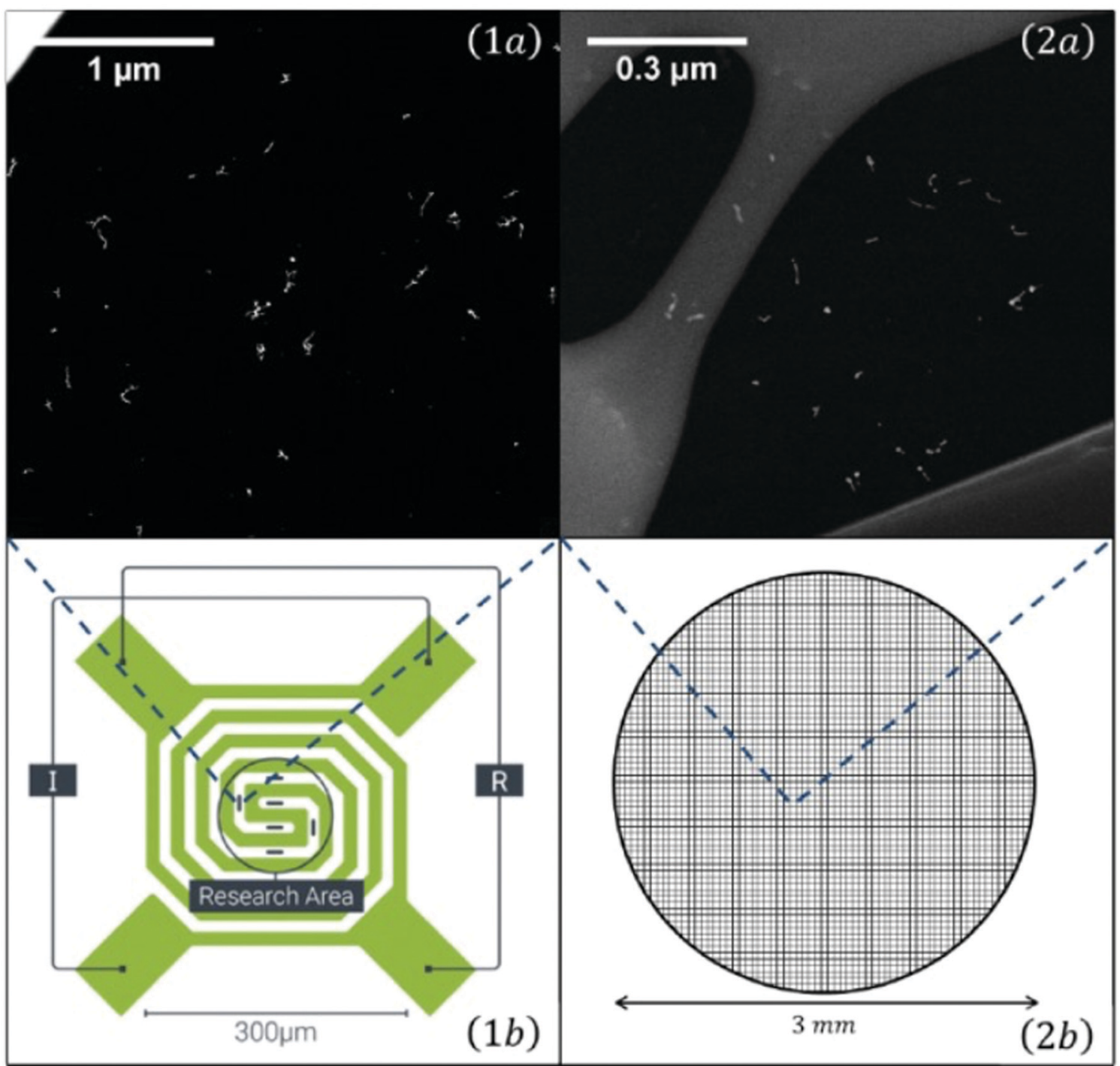

Fig. 5 Overview of the two different transmission electron microscopy (TEM) grids used for temperature dependent measurements: On the left a heatable carbon TEM grid (1) (DENSsolutions Nano-Chip XT carbon) which is able to provide a temperature range between room temperature and $1300{ }^{\circ} \mathrm{C}$. The carbon windows are embedded inside a heatable filament. The second grid (2) is an amorphous carbon grid used for cryogenic experiments, ranging from $-150{ }^{\circ} \mathrm{C}$ up to room temperature. A close up view of the two different carbon windows can be seen in the upper two panels (1a and 2a). It is easy to identify the difference between the larger, but less numerous windows of the heatable grid and the amorphous carbon grid, which consists out of several smaller windows. Taken from ref. 79.

A comparison of the results of computer modeling the cluster landing, ${ }^{76}$ the atomic resolution electron tomography, ${ }^{78}$ and an intensity based evaluation of HAADF images let us conclude that the originally formed cluster shape inside the droplet is only weakly altered upon deposition. Further comparison of the cluster size distribution measured by TOF-MS in the beam with that on the TEM grids indicates that the clusters do not break up. ${ }^{55}$

For optical absorption measurements of cluster samples, fused silica microscope slides for deposition and a Shimadzu UV-1800 spectrophotometer have been employed. ${ }^{63}$ Plasmon resonances of metal clusters obtained this way, represent an average over a certain size distribution, but significant differences for single metal as opposed to core shell particles were found. Furthermore, such ensemble averaged measurements were conducted for surface enhanced Raman studies. ${ }^{63}$ Plasmon resonances for individual nanoparticles on a thin h-BN substrate, which provides very low background absorption, could be studied with EELS. ${ }^{75}$

Atomic Force Microscopy (AFM) required exposure to air and was only applied to Ag@Au core@shell particles on fused silica (see Fig. 6). Comparison of the AFM scans was made with an image obtained with an Environmental Scanning Electron Microscope (ESEM) such as to test the compatibility of the prepared sample with a real world environment. For a high surface coverage, the core-shell nanoparticles were synthesized in a strong helium droplet flux generated by cooling the nozzle to $5 \mathrm{~K}$. Under these conditions, the particles are nanowire-like with elongated shapes due to the presence of vortices in the droplets. A surface coverage of about $20 \%$ is reached in about $15 \mathrm{~min}$, while the production using a nozzle temperature of about $9 \mathrm{~K}$ yields more uniform spherical metal particles but only about $9 \%$ 

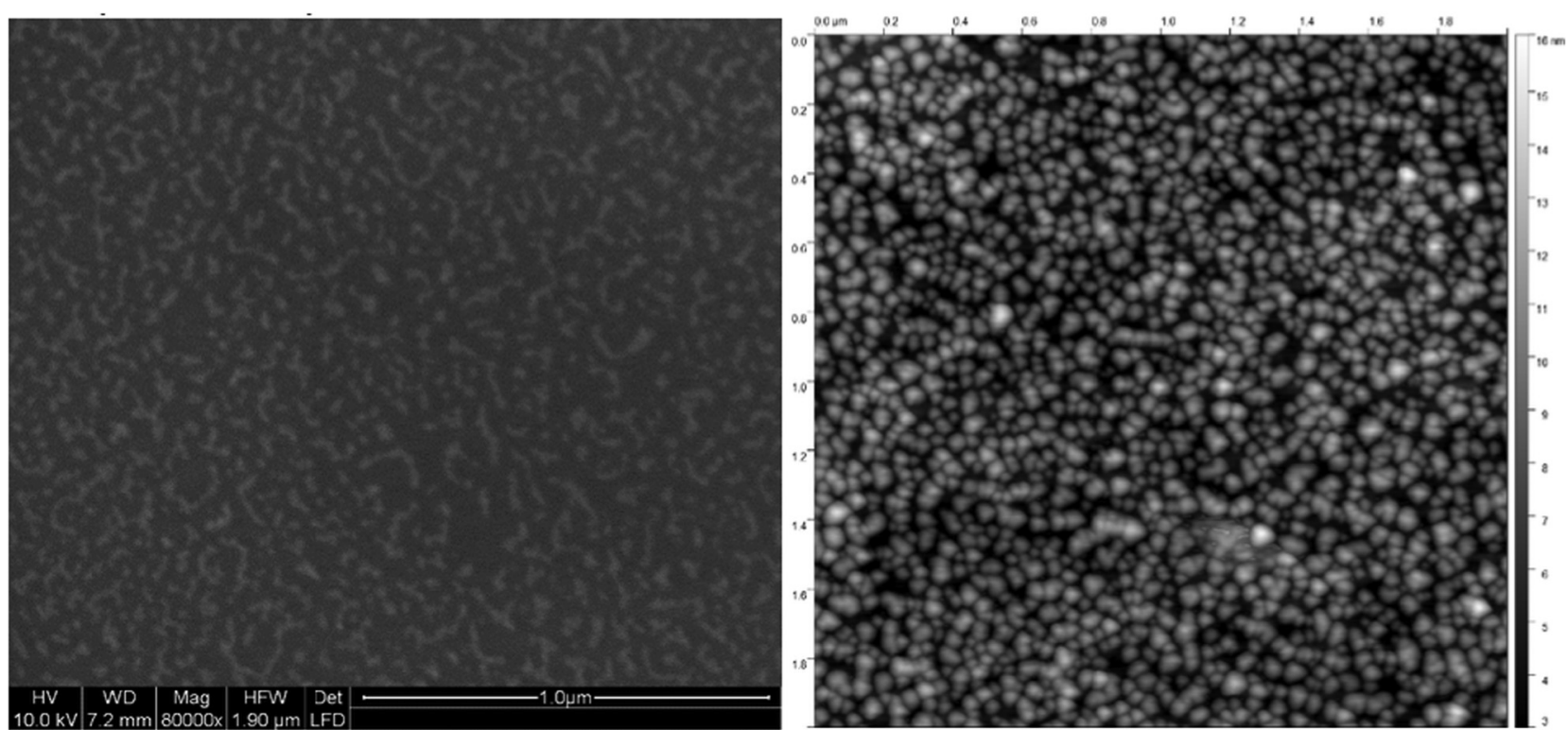

Fig. 6 Ag@Au core@shell nanoparticles of elongated shape, similar to ref. 60, imaged with an ESEM at only $10 \mathrm{~nm}$ resolution (left panel) compared to an AFM image in the tapping mode with an undetermined tip size (right panel). The scale is the same with the total area being $2 \mu \mathrm{m} \times 2 \mu \mathrm{m}$. The coverage is estimated as $26 \%$ based on the ESEM image while the AFM gives the impression of a higher coverage. ${ }^{80}$

coverage per hour. A comparison of low resolution electron microscopy and AFM images gives the impression that the AFM leads to an overestimation. In the depicted case, the AFM was operated in the tapping mode and no effort was made to optimize the tip size. We believe that the resolution is even worse than in the ESEM recording and that the particle shapes are not reproduced correctly but may have been influenced by the tip.

For STM studies, gold clusters were deposited on HOPG to provide enough conductivity, but the cluster mobility on this substrate turned out too high leading to unwanted new cluster patterns. ${ }^{81}$

With the recently acquired NanoESCA (Scienta Omicron), energy resolved photoemission electron microscopy has become possible. By illumination with a helium lamp $(21.2 \mathrm{eV})$, a first workfunction mapping of a $8.6 \mu \mathrm{m} \times 8.6 \mu \mathrm{m}$ field of view of the pristine p-type silicon substrate compared to the same substrate covered with $\mathrm{ZnO}$ nanoparticles was obtained (see Fig. 7).

\section{Theoretical methods for modelling cluster aggregation and thermally induced dynamics}

\subsection{Metal cluster formation}

Theoretical descriptions of metallic cluster aggregation processes inside He droplets must either employ reasonable estimates of atomic collision probabilities or try to model the actual dynamics of the immersed impurities at a molecular level. Attempts have
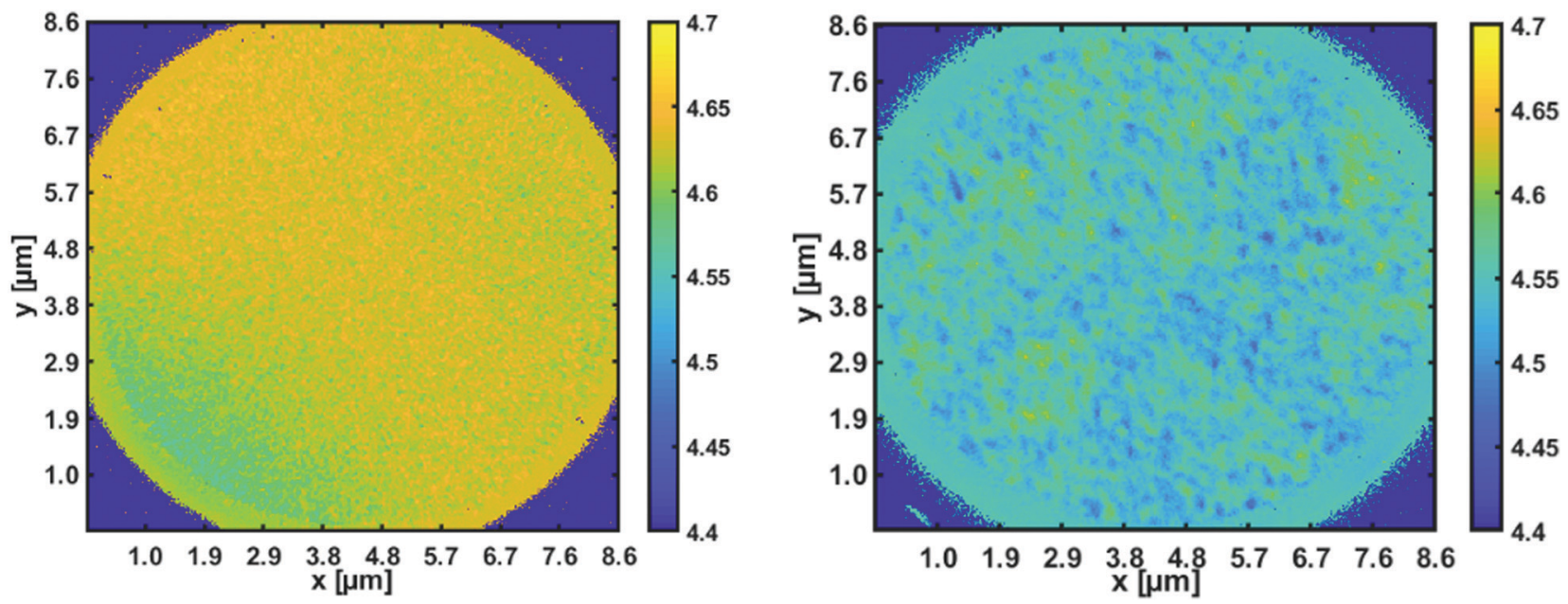

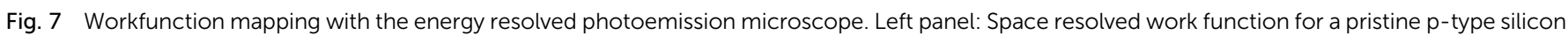
wafer [4.7 eV]; right panel: reduction to about $4.5 \mathrm{eV}$ in areas covered with ZnO nanoparticles. ${ }^{82}$ 
been made in both directions, but with different intentions and scopes due to the inherent complexity of the matter.

Accurate descriptions of He droplets with sizes in the range of several thousand He atoms and a few impurity molecules or atoms are currently achieved via Helium Density Functional Theory (He-DFT), ${ }^{83,84}$ most effectively through the BarcelonaToulouse program package. ${ }^{85}$ This powerful open source software tool allows the simulation of metallic impurities together with quantum vortices, ${ }^{21,86,87}$ an interesting phenomenon which affects cluster formation in larger He droplets. While interactions with the desired impurities are introduced by summations over effective two-body potentials, the actual quantum behavior of the surrounding $\mathrm{He}$ is nicely captured with this technique. In more recent time-dependent studies, even the coupling between helium density fluctuations, i.e. the 'nuclear part' of the total wavefunction, and the electronic excitation of single impurity atom inside the helium droplet has been simulated successfully in order to interpret femtosecond pump-probe spectroscopy measurements. ${ }^{46}$ Of further interest is the interaction between impurities and vortices, which are known to exert an attractive 'pinning' force on the letter. ${ }^{58,83,88}$ Although this circumstance is crucial (as will be detailed below) for certain metal cluster formation processes, the still ongoing and fruitful fundamental research on superfluid helium dynamics still resides almost fully disjoint from the actual experimental research in the field of helium-mediated metal cluster synthesis. The reason for the discrepancy lies in a certain limit of the He DFT approach with regards to the emulation of pronounced collective excitations and very large deformations produced by strongly interacting or very fast impurities. ${ }^{88,89}$ Also, the rather crude ansatz of pair-sum potentials does only hold for certain cases. Yet, the fascinating insights gained by He-DFT can currently not be delivered by any competing method such as e.g. full $a b$ initio calculations, path integral molecular dynamics ${ }^{90}$ or quantum Monte Carlo treatments $^{91,92}$ (such as Green's function Monte Carlo or diffusion Monte Carlo methods) of the systems at hand due to the high computational cost of these alternatives which forbid their application in size regimes relevant for metal cluster growth. However, these techniques have been successfully applied to smallest, helium-immersed metallic clusters of $\mathrm{Na}$ and $\mathrm{Mg},{ }^{93,94}$ partially aiming to answer the long-ongoing debate concerning the existence of metastable, foam-like metallic structures formed by weakly interacting metal atoms. ${ }^{95}$

A less refined but effective strategy to bridge this gap between simulation capabilities and current experimental research was based on the combination of classical Newtonian molecular dynamics for a given impurity with He DFT calculations to obtain estimates for the retaining force which acts on it and keeps it inside the He droplet. ${ }^{56}$ This technique was easily extended to droplet sizes which were realistic for metal cluster formation, and could be used to derive approximate collision times for two metal atoms immersed into a helium droplet. By comparing the latter to averaged pickup rates, tentative estimates of the actual 'growth' mechanism inside the droplets could be made, as the process switches from single- to multicenter growth if the pickup rate exceeds the average collision time. Via time evolution studies, where the number and mass of all metal particles was tracked over time (but not their actual position), a time-dependent distribution of metal particle sizes could be obtained for time intervals in the range of milliseconds. ${ }^{49}$ These simulations also took the evaporation of He due to collision energy dissipation into account, which leads to a shrinking of the actual droplet size over time, and even allowed the implementation of vortex pinning effects via an effective correction based on averaged times for impurity trapping in a single, simplified (i.e. straight) vortex line. Inclusion of the latter effects turned out to be crucial for the explanation of metallic nanowire formation as it had been observed in numerous experiments, ${ }^{59-62}$ since the impurity-collecting nature of vortex lines enforces a preferred one-dimensional growth of metallic structures in the form of irregularly shaped nanowires. An interesting finding of these simulations was that these wires are formed by a coagulation of larger metal clusters, baked together through the pinning effect, but without a complete melting upon contact. ${ }^{49,96}$ Therefore, the morphologies of the initial building blocks, i.e. the interfaces of spherical clusters, are preserved in the final structures, as was seen in TEM images with atomic resolution. Examples will be shown in Section 5 .

\subsection{Deposition and restructuring effects}

Between the growth of a metallic structure inside a He droplet and its final imaging on a suitable substrate lies the crucial process of its preferably non-invasive deposition. As it turns out, the deposition of loaded He droplets can truly be considered a non-damaging type of soft-landing, no matter the size of the actual impurity, but for different reasons.

In the case of single metal atoms, the helium embedding plays a crucial role as it facilitates a sticking probability of almost one and a negligible delocalization of the impurity atom upon impact, as was shown for the case of He-immersed $\mathrm{Au}$ atoms on $\mathrm{a} \mathrm{TiO}_{2}$ surface. ${ }^{77}$ This original study used (electronic) density functional theory to evaluate the interaction potential of a single He atom with bulk titanium oxide and used this information in follow-up simulations of the impact dynamics via timedependent He density functional theory, allowing for a realistic treatment of the helium environment as a quantum fluid. In contrast to classical simulations, a time evolution of the bosonic, macroscopically extended helium droplet wavefunction showed a continuous, well behaved spreading of the helium over the bulk surface instead of any chaotic splashing behavior.

In a second article of the same research group the deposition of larger silver clusters (consisting of up to 5000 atoms) on amorphous carbon and on graphene was investigated, ${ }^{97}$ this time via molecular dynamics simulations only, but with more complex interaction potentials carefully derived from preliminary ab initio studies. Interestingly, these simulations reveal that even bare $\mathrm{Ag}$ clusters can be deposited without damaging (if impact velocities and cluster temperatures of He-immersed species are assumed) due to their ability to temporarily deform and to dissipate the impact energy without considerable structural changes. In the larger size regime, the soft-landing is therefore more 'assisted' than enabled by the He droplets. A more pronounced effect of the 
helium immersion was discovered regarding the actual distribution of clusters on the support after deposition, suggesting that secondary impacts play a larger role than surface migration of clusters residing already on the surface.

A third and final look at this fascinating step in metal cluster production is concerned with cluster sizes in between these two extremes mentioned above. Ref. 76 was dedicated to the study of deposition effects on silver clusters of different morphology (icosahedral, dodecahedral and face centered cubic) in the range between 100 and 2000 atoms as a function of the landing conditions, using the Sutton-Chen potential for metallic interactions and a Morse potential for the surface, with its parameters chosen to emulate an unstructured, rigid carbon model surface. While this study confirms the stability of larger clusters which remain mostly unaffected by the impact, its also predicts structural transitions in smaller clusters for certain landing conditions. A clear tendency to restructure into a single domain close-packed morphology is noted and in good agreement with structure statistics derived from experimental findings. The occurrence of a structural transition seems to depend on the initial structure, suggesting that decahedral clusters tend to conserve their morphology better than icosahedral ones.

\subsection{Metal structure stability}

The ability to grow and deposit metallic nanowires triggered a series of detailed studies on their structural features after deposition. Particularly disturbing was the experimental finding for silver, which did not form wires but 'pearl chains' of small droplets, which caused vivid discussions in the community regarding potentially different growth scenarios for different metals inside the He droplets or a possibly destructive deposition. The actual reason for this exceptional behavior could be revealed in ref. 98, after the successful deposition of intact silver structures onto a sample holder which had been cooled down to $77 \mathrm{~K}$. At this temperature, and almost up to room temperature, silver also shows the expected formation of wires with diameters in the range of about $5 \mathrm{~nm}$, but starts to decompose at higher temperatures due to Rayleigh breakup. This phenomenon, a well-known effect in fluid dynamics, where the growth of initial diameter perturbations leads to the disintegration of the respective jet, is driven by diffusion of metal atoms on the wire surface, attempting to minimize their energy by a relocalization away from thinner areas with larger curvatures toward less strained cluster regions. As a consequence, 'dents' in the irregularly shaped wires tend to become more and more constricted until a full breakup into separate parts is established. Computationally, this behavior was reproduced at first through a two-dimensional model, employing a relation ${ }^{99}$ between the local atomic diffusion tendency and the actual curvature of a piece of wire at this particular point. ${ }^{98}$ TEM images of intact nanowires, effectively two-dimensional projections of the actual structures, were translated into closed contour loops which served as initial structures for the numerical integration of a resulting partial differential equation. This way, time evolutions of the contour shape were obtained, which also included breakups, emerging in this treatment as the formation of two new closed loops produced from the original one by constriction. While this method could correctly predict structural changes over time, it suffered from a lack of computational practicality, in particular when applied to complicated, more realistic and better resolved initial contour lines or in time evolutions of several wires simultaneously. For this reason an alternative computational concept based on cellular automata had been developed. ${ }^{100}$ In this ansatz, the actual distribution of material is approximately reconstructed from TEM images and translated into a threedimensional grid model. Each cubic cell of this model, called a voxel, represents approximately 55 to 85 metal atoms if filled with material. The determining feature of a cellular automaton is that the current state of a voxel, i.e. filled or empty, is updated in a periodic fashion, but only dependent on the states of its neighboring voxels. In a series of time steps, each one corresponding to a state update of all cells or voxels in the model, the migration of metal atoms is enabled through update rules, here realized by a Monte Carlo method deciding whether a flow of material from one voxel to a neighbor voxel is taking place or not. This approach turned out to be much more robust and less costly compared to the contour-based method and could be successfully applied to real $\mathrm{Ni}, \mathrm{Cu}, \mathrm{Ag}$ and $\mathrm{Au}$ nanowire structures. ${ }^{101} \mathrm{It}$ allowed the prediction of wire breakup positions as well as their correct order of appearance in time upon heating.

\subsection{Structural behavior of mixed-metallic clusters}

A last topic to be addressed in the theoretical part of this review is the description and simulation of structural behavior of mixed-metallic structures after deposition onto a given substrate. Numerous, wellestablished methods are applicable to this objective in principle, but the final choice is mostly determined by the system size and the accuracy required to test a certain hypothesis via a computational experiment. With this being a very general problem of cluster physics, and fully independent of any specific route chosen for the synthesis of the desired metal structures, even an attempt to briefly summarize current developments in this field would be misplaced in the scope of this much more specific review. Instead, after referring to a few more recent, excellent overviews on the theoretical treatment of (mixed-)metallic clusters, ${ }^{102-106}$ we present and discuss some concrete attempts addressing the peculiarities of He-grown, mixed-metallic structures.

Obviously, all of these peculiarities are a consequence of the still not fully understood growth mechanisms inside a $\mathrm{He}$ droplet. The cold and fully inert environment suggests an undisturbed, preferably spherical growth of a metallic cluster in its structural configuration of lowest energy, presuming that the rate of metal atom pickup and particle agglomeration are set up accordingly through appropriate choices of He droplet size and vapour pressure in the pickup chamber. For monometallic clusters, this specific way of synthesis is not expected to produce structures which differ significantly from those created by any other method, as long as the inevitable external influences (templates, seeds, precursors and solvents) which compromise the latter are kept at a minimum. However, for metallic cluster compositions consisting of two or more elements, the nature of the experimental setup enforces a highly specific growth 
in the form of spherical layers. The resulting onion-type structures, in their simplest form core-shell clusters, have been studied in great detail recently. ${ }^{45,63,64,105,107-111}$

For cluster sizes in the range of several thousand atoms a computational treatment with advanced methods of electronic structure theory is not feasible, at least not the metallic structure as a whole. On the other side, meso-scaled approaches such as the coarse-grained Monte Carlo approach discussed above are incapable of describing diffusion and alloying processes which take place on the atomic scale. This leaves the application of suitable force fields as the only choice, which are typically based on the embedded atom model. ${ }^{112}$ The latter ansatz includes the pair energy between two atoms as well as contributions stemming from the embedding of each metal atom into its local environment. In our recents works, ${ }^{111}$ we employed potentials of the Zhou group for $\mathrm{Au}^{113}$ and for the iron triade elements Fe, Co and $\mathrm{Ni}^{114}$ which are of the quantum Sutton-Chen type. ${ }^{115,116}$ Attempts have also been made to build improved force fields via machine learning methods such as Gaussian regression or artificial neural networks. ${ }^{117,118}$

When used in large-scale molecular dynamics simulations, preferably run in parallel on supercomputers, molecular dynamics simulations can be performed to study the structural behavior of finite, mixed-metallic structures at various temperatures. With methods similar to those described in ref. 111 and 119, even the process of cluster formation inside the droplet can be (at least very rudimentarily) emulated by a certain choice of metal atom spawning followed by heating and cooling to allow for a continuous structural minimization during particle growth. The same methods also allow for investigations of metal alloying, at least in the onset, but are limited by the unfeasible time lengths needed to simulate the experimentally observed diffusion processes in the range of several nanometers. This problem can be overcome by less realistic but more efficient sampling techniques, preferably on a discrete grid, where single atom motion is fully replaced by artificial, temperature-dependent hopping moves or 'atomic swaps' within a Monte Carlo approach. ${ }^{111}$ After each artificial move a structural minimization is performed. This way, a much larger portion of the accessible state space can be sampled, however at the cost of no longer simulating real transitions from one geometry to the other. In this simplified picture, even free energies can be estimated as a function of temperature, since the configuration entropy, i.e. the portion of the total entropy stemming from the multitude of possible arrangements (e.g. $n$ atoms of type $\mathrm{A}$ and $m$ atoms of type $\mathrm{B}$, given $n+m$ possible lattice positions), is the leading contribution to the total entropy. ${ }^{105}$ For largest metallic clusters, where even the crude, yet still atomistic lattice models reach their computational limit, empirical corrections of the well-known bulk phase diagrams can be employed, e.g. the CALPHAD method ${ }^{120}$ in combination with a $1 / r$ correction to account for the increasing surface to volume ratio with decreasing cluster radius $r .{ }^{121}$

In the case of surface reactions such as e.g. the simple oxidation of one metal component, the application of electronic structure methods becomes inevitable, although tentative 'reactive' force fields have been suggested and tested for selected reactions. ${ }^{122}$ Typically, these calculations are based on density functional theory, employing plane wave basis sets in a periodic setup. Small slabs, i.e. representative areas of the material, comprising several unit cells of the actual bulk metals, are used to simulate either inner regions of a given mixedmetallic cluster, e.g. to study the interface of a certain core-shell structure, or regions of the cluster surface, e.g. to investigate reactions with gas molecules. On the downside, although local features such as surface reactions on active centers can be nicely simulated, alloying or diffusion behavior on a larger scale can barely be captured by these accurate but computationally very expensive methods.

\section{Results}

In this section, we present a selection of results on metal clusters synthesized in helium droplets that show the high potential of this method for the investigation of fundamental properties of nanoparticles undisturbed by residuals from chemical preparation.

\subsection{Metallic nanowires and their thermal stability}

In the late 1940's, Onsager put forward the idea of quantized circulation in superfluid helium which was followed up by other low temperature physicists like Feynman who predicted details about the related vortex cores. ${ }^{86}$ Visualizing quantum vortices in an experiment took a long time since their discovery until Bewley, Lathrop, and Sreenivasan could photographically image the hydrogen particles that had accumulated along vortex cores in their liquid helium cell. ${ }^{123}$ Shortly after this, Gordon and coworkers injected a hydrogen gas jet into a cryogenically cooled helium II bath and observed the formation of narrow filaments of hydrogen as traces of vortex lines. ${ }^{124}$ Gordon and coworkers together with Weiss and Moroshkin followed this path and created metallic filaments inside bulk superfluid helium taking advantage of the attracting forces of the vortex cores, ${ }^{125,126}$ deposited nanowire networks on substrates by evaporation of the liquid helium, and even measured the electric conductivity of such nanowire bundles. ${ }^{127}$

Vilesov and coworkers doped large helium droplets produced at low nozzle temperatures, with silver atoms. After deposition on a solid substrate they recognized arrays of silver clusters that lined up like an interrupted nanowire. ${ }^{59}$ Those traces were interpreted as first evidence of vortices in helium droplets. Similar broken chains of Ag clusters were observed by Spence et $a l .{ }^{61}$ but there was no explanation why the $\mathrm{Ag}$ clusters would not have grown together.

Suspecting the breakup being an effect of the room temperature substrate and as such happening after the deposition, we terminated the Ag-doped helium droplet beam on a liquid nitrogen cooled amorphous carbon substrate. ${ }^{98}$ Furthermore, we conducted similar experiments under the same $\mathrm{He}_{N}$ source conditions with $\mathrm{Au}, \mathrm{Cu}$, and $\mathrm{Ni}$ dopant atoms. ${ }^{101}$ For room temperature deposition, Fig. 8 shows the metal cluster traces for $\mathrm{Ag}$ (left panel), $\mathrm{Au}$ (center panel) as well as $\mathrm{Cu}$ and $\mathrm{Ni}$. 

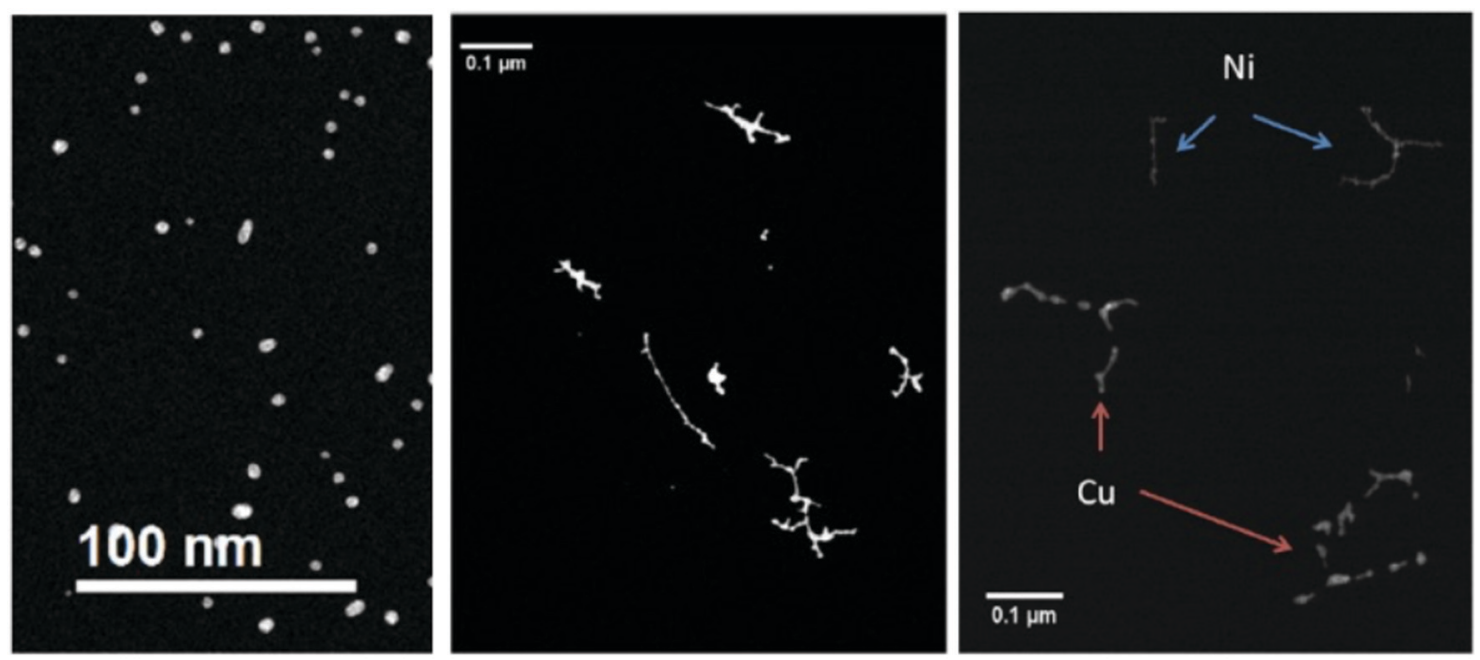

Fig. 8 Four different nanowire materials studied at room temperature. All of them are synthesized at $T_{\text {nozzle }}=5.4 \mathrm{~K}$ and with $\alpha=0.75$. In the left panel the thermally very unstable silver nanowires are already fully patternized, as a result of a very low energy barrier towards starting the surface diffusion process. In the central panel the noble metal gold is illustrated. Gold is not only stable at room temperature, but it also shows no or nearly no oxidation at its surface. As a result, gold is an ideal candidate to study the unperturbed phenomenon of surface diffusion. The non-noble metals nickel and copper are presented in the right panel. Both of them are covered with an oxide layer due to the vacuum conditions in the electron microscope, which effectively stabilizes the synthesized nanowires. From ref. 79.

Obviously, other metals than silver yielded uninterrupted cluster chains or nanowire like structures.

Deposition on a liquid nitrogen cooled substrate revealed the survival of a vortex induced $\mathrm{Ag}$ nanowire formation even after deposition. As depicted in Fig. 9, the uninterrupted chain is stable up to $253 \mathrm{~K}$ and then starts to disintegrate at higher temperature. $\mathrm{Au}$ nanowires can be heated much higher before they fall apart at about $573 \mathrm{~K}$. This different behaviour can be seen in the electron microscope images in Fig. 10, which are shown in panels a and b. Panels $\mathrm{c}$ and $\mathrm{d}$ are model pictures derived from the cellular automaton approach explained in the previous section.

Because of the large size of the nanowire system, the model calculation of the observed Rayleigh breakup uses cell sizes of a volume that corresponds to the resolution of the transmission electron microscope images. As shown in ref. 100 and 101, the simulations are in good agreement with the experimental observations for $\mathrm{Ni}, \mathrm{Cu}, \mathrm{Ag}$, and $\mathrm{Au}$ nanowires of about 2 to $5 \mathrm{~nm}$ diameter. The differences in the behaviour of the different metals are expressed by an equation connecting the nanowire breakup temperature with the corresponding bulk metal melting temperature, the nanowire diameter and a metal specific constant being parameters. The breakup itself, however, is purely due to surface diffusion and does not require melting. In Fig. 11, the simulated temporal evolution of the diffusion process leading to breakup is compared with a sequence of electron microscope images for a gold nanowire structure kept at $423 \mathrm{~K}$.

Depending on the doping rate of the helium droplet beam, the nanowire growth along a vortex line leads to longer or shorter structures. In ref. 49 the doping rate is expressed by a parameter $\alpha$ between 0 and 1 that gives the attenuation of the $\mathrm{He}_{N}$ beam arriving at the QMS at the end of the beam apparatus. For example, $\alpha=0.75$ ('heavy doping') means that the attenuation amounts to $75 \%$, and the large amount of metal atoms loaded on to a helium droplet caused strong evaporation of helium. Our simulation of the initial multicenter growth of spherical metal clusters via the collisional model of ref. 55 and subsequent attraction into the vortex core yields the trapping times listed in Table 1 (see also the correction of ref. 49 in Phys.
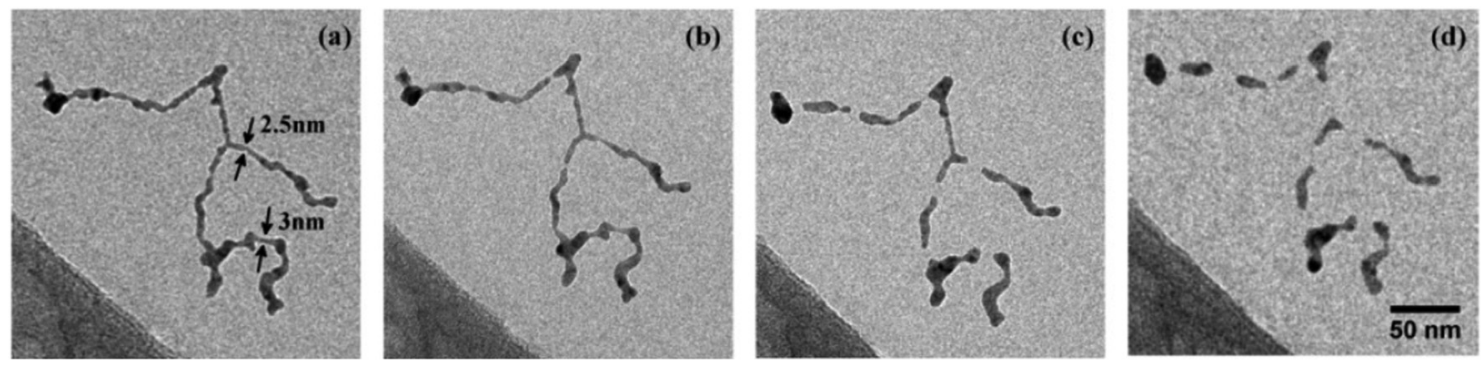

Fig. 9 Structure evolution of a Ag nanowire at increasing temperatures of $253 \mathrm{~K}$ (a), $268 \mathrm{~K}$ (b), $293 \mathrm{~K}$ (c), $363 \mathrm{~K}$ (d). Structure as deposited (no change to situation at $77 \mathrm{~K}$ ) is shown in (a). (b) Marks the begin of wire breakup after surface smoothing, while (c) shows the fully segmented wire at ambient conditions. At higher temperatures the relaxation into spheres is suppressed by a carbonaceous capping layer (d). Scale is the same for all images. Ref. $98-$ reprinted with permission of the PCCP Owner Societies. 


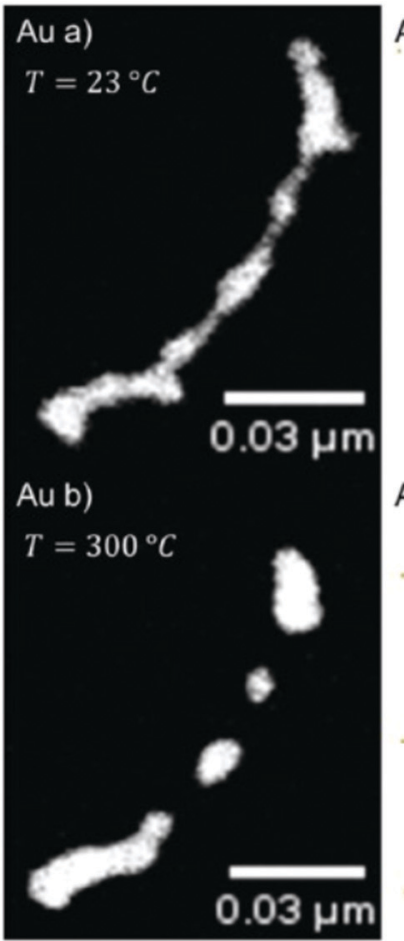

Au c)

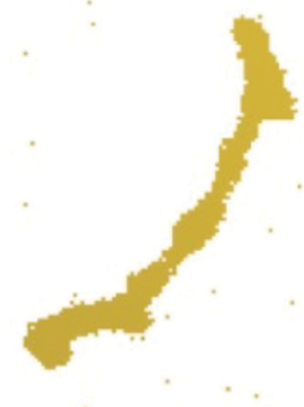

Au d)

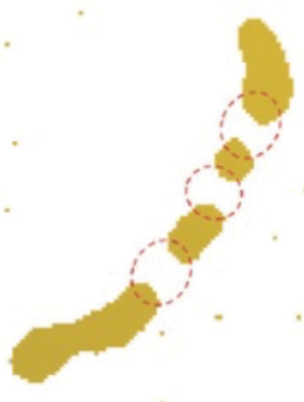

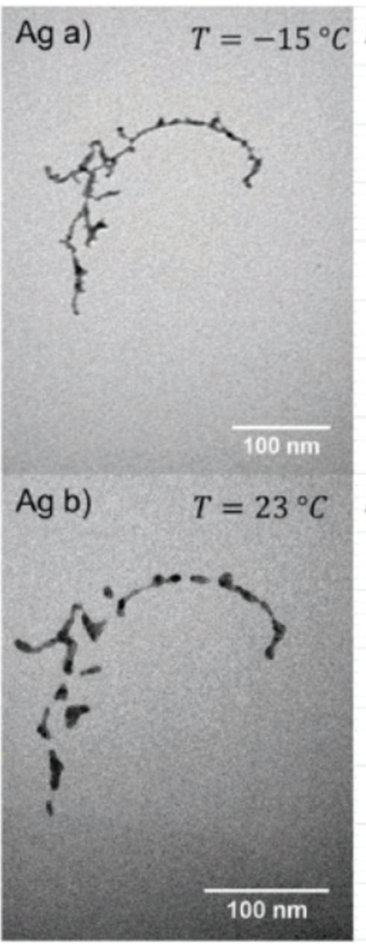

Fig. 10 Comparison between measured surface diffusion processes and cellular automaton prediction of Au and Ag nanowires. In (a and c) one can see the initial structure and the converted structure used in the cellular automaton approach, respectively. Those structures evolve to the ones shown in (d), when heating is applied, in very good agreement with the final structures observed experimentally (b). Ref. 101 - reprinted with permission of the PCCP Owner Societies.

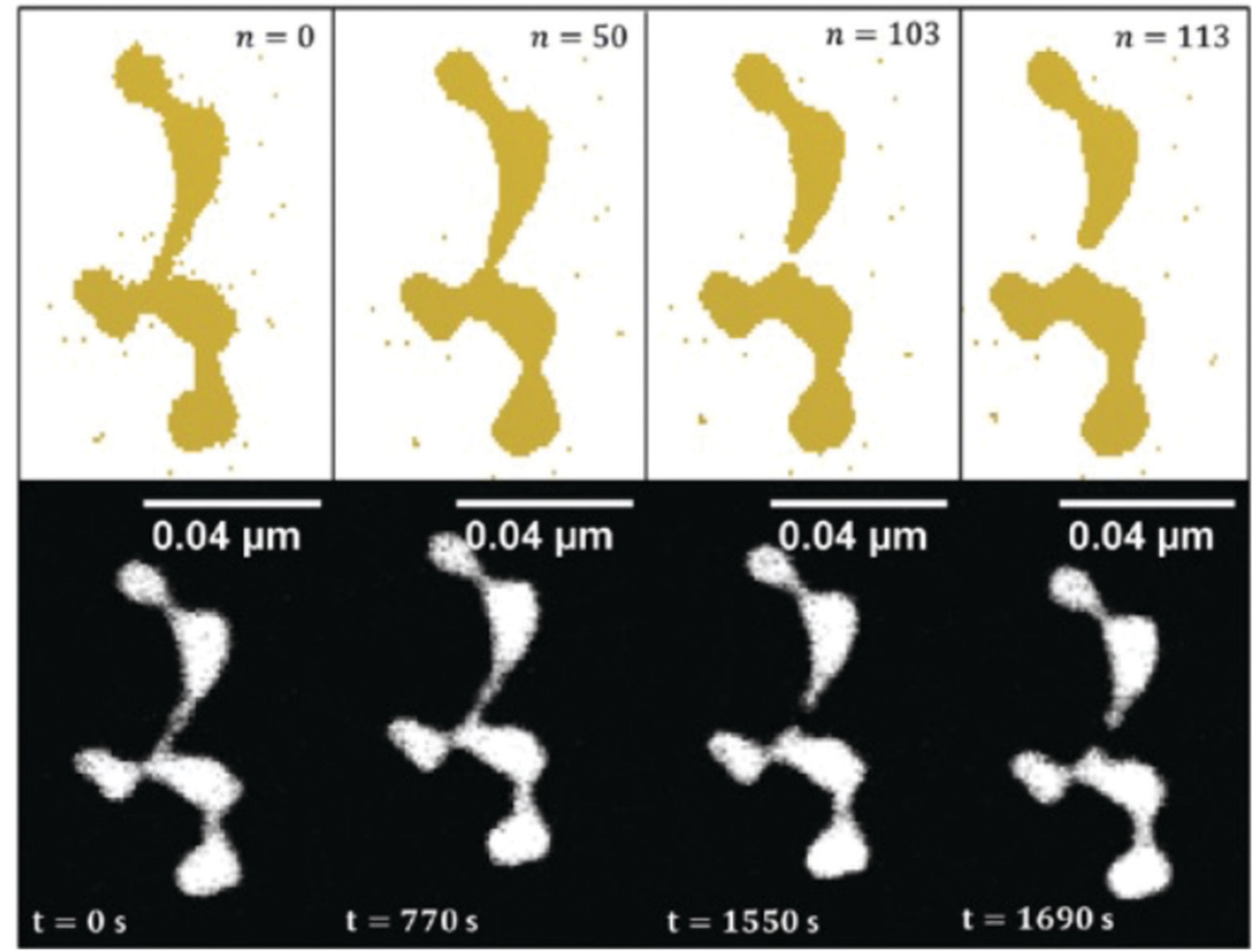

Fig. 11 Time evolved surface diffusion process of a gold nanowire at $150{ }^{\circ} \mathrm{C}$. The observed in situ breakup event is studied over a time period of nearly half an hour (lower images) and are compared to the results obtained with the CA (upper images) in order to draw a fit between iteration steps and experimental observation time. Ref. 101 - reprinted with permission of the PCCP Owner Societies. 
Table 1 Time $t_{\text {trap }}$ until a particle gets trapped by a vortex due to the attractive force. The final droplet diameter $d_{\mathrm{D}, \alpha}$ is calculated assuming an initial $\mathrm{He}_{N}$ size of $1.7 \times 10^{10}$ atoms, taking the doping-dependent evaporation of helium atoms into account. For more details, see ref. 49 and its correction in Phys. Chem. Chem. Phys., 2016, 18, 3359

\begin{tabular}{llrl}
\hline Element & $\alpha$ & $d_{\mathrm{D}} / \mathrm{nm}$ & $t_{\text {trap }} / \mu \mathrm{s}$ \\
\hline $\mathrm{Ag}$ & 0.75 & 720 & 24 \\
& 0.20 & 1060 & 52 \\
$\mathrm{Au}$ & 0.75 & 720 & 33 \\
& 0.20 & 1060 & 71 \\
& 0.04 & 1130 & 80
\end{tabular}

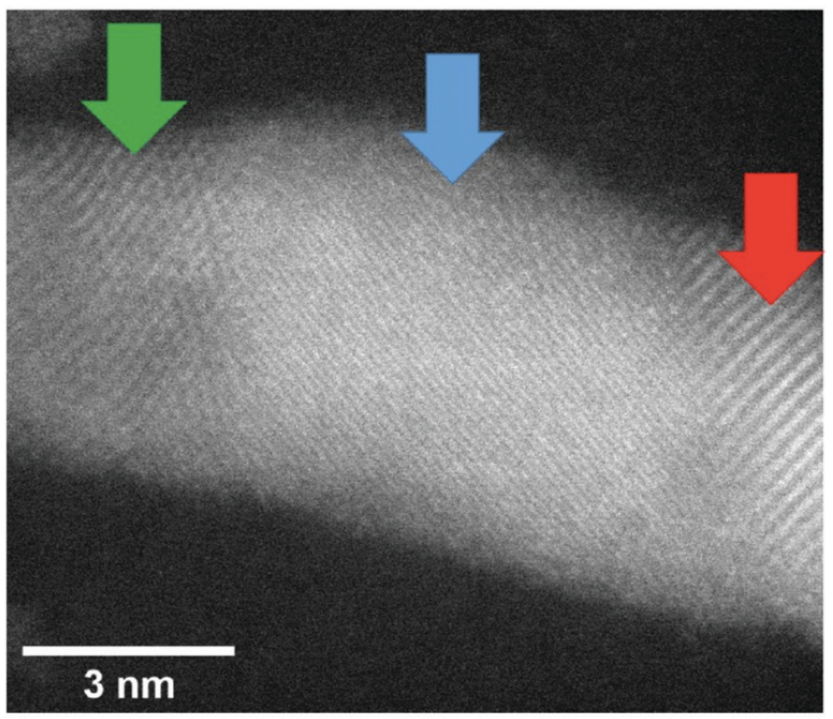

Fig. 12 Image of a Au nanowire portion in which the lattice structures are shown. Three different regions were evaluated: (red) a lattice distance of $0.24 \mathrm{~nm}$ was measured indicating the (111) surface $(0.234 \mathrm{~nm})$, (blue) lattice distance of $0.12 \mathrm{~nm}$ was measured which may correspond to a (222) $(0.117 \mathrm{~nm})$ lattice and (green) a lattice distance of $0.20 \mathrm{~nm}$ was measured indicating the (200) $(0.2035 \mathrm{~nm})$ lattice. Note that for the blue region also different lattice assignments would be possible. The error of this measurement is $\approx 0.01 \mathrm{~nm}$. From ref. 96 .

Chem. Chem. Phys., 2016, 18, 3359). For comparison, individual metal clusters of 1000 atoms are formed already after $\approx 1 \mu \mathrm{s}$ while the vortex trapping times are more than 10 times longer.

A close-up of the structure of a gold nanowire near the seam between the clusters lining up along a vortex line is depicted in Fig. 12 and reveals the changes of the lattice geometry like in a statistically grown connection.

While the results of detailed investigations of core@shell clusters are presented in the next section, it is worth mentioning that long core@shell nanowires, formed by weakly connected clusters, clearly exhibit the character of a chain of individual core@shell clusters rather than having a long well connected core with a uniform coating over the full length. ${ }^{60}$ This can be understood when considering the sequential growth process explained above.

\subsection{Core@shell particles and alloying}

With our setup as depicted in Fig. 1, almost any core@shell nanoparticle of an atomic or molecular composition can be synthesized if the constituents can be provided in a pickup cell at a vapour pressure of $10^{-5}$ to $10^{-4} \mathrm{mbar}$. Due to the helium mediated soft ionization, ${ }^{67-69}$ the TOF-MS shows the element specific loading of the droplet. The ultrahigh vacuum (UHV) in the deposition chamber guarantees almost contamination-free samples. UHV-transport is facilitated by the Ferrovac Vacuum Suitcase (see Section 3). Oxidation of highly reactive metals can occur when the vacuum conditions cannot be maintained. For example, our own experiments with deposited chromium clusters on the quartz microbalance with $10^{-6}$ mbar rest gas in the chamber have shown an enormous mass increase over a time interval of 2 hours. It can be estimated that under these 'high vacuum' conditions a sample with chromium clusters will be fully oxidized in a day. This experience motivated us to start coating reactive metal clusters with an inert metal like gold. Such core@shell metal clusters exhibit additional advantages if they provide interesting combinations of material properties like plasmonic resonances, magnetism, or semiconductor features paired with a metallic core.

First experimental studies concentrated on $\mathrm{Ag}-\mathrm{Au}$ combinations because they provided clean nanoscale structures without any impurities. Fig. 13 shows a detailed structural and elemental analysis via electron microscopy methods. ${ }^{78}$ The clusters stay intact and subsequent work concentrated on the questions of thermal stability and phase changes towards nanosized alloy formation. Transmission electron microscopy images of Ag@Au and Au@Ag core@shell particles were recorded for sample temperatures from room temperature up to $300{ }^{\circ} \mathrm{C}$ at which the clusters appeared fully alloyed while a crystalline structure was resolved over the full temperature range. ${ }^{43}$ The mixing process was therefore interpreted as solid state diffusion. Density profiles for the core and shell materials were determined from the images and a diffusion model with a temperature dependent effective diffusion constant for the $\mathrm{Ag}-\mathrm{Au}$ nanomixture presented. The results were compared with an existing phase diagram for the AgAu system as a function of the particle radius. It turned out that in the size range of our clusters, mixing occurs already at about 200 to $300 \mathrm{~K}$ lower temperature than previous scaling laws would predict. We conclude that the diffusion on this length scale (below $5 \mathrm{~nm}$ ) is initiated at lower temperatures than in the corresponding bulk material, which is a consequence of dominant surface size effects in this regime. For both systems (Ag@Au and Au@Ag), alloying takes place between 500 and $550 \mathrm{~K}^{43}$

The elements $\mathrm{Fe}, \mathrm{Co}, \mathrm{Ni}$ (also known as iron triade) provide a magnetic moment which makes them interesting candidates for nanosensor applications. Because of their reactivity, a gold coating appeared attractive as passivation layer. ${ }^{111}$ Experiments with different Au-shell thickness had revealed that 3 atomic gold layers provide a minimum protection of a $2 \mathrm{~nm}$ diameter Fe-core. ${ }^{64}$ A similar protection layer is necessary for a Ni-core under normal environmental condition. Heating experiments, however, showed that a cycle of heating up to $400{ }^{\circ} \mathrm{C}$ with subsequent cooling to room temperature in the presence of small traces of oxygen resulted in a core-shell inversion with nickel oxide outside a gold core. ${ }^{109}$ The influence of oxygen on 

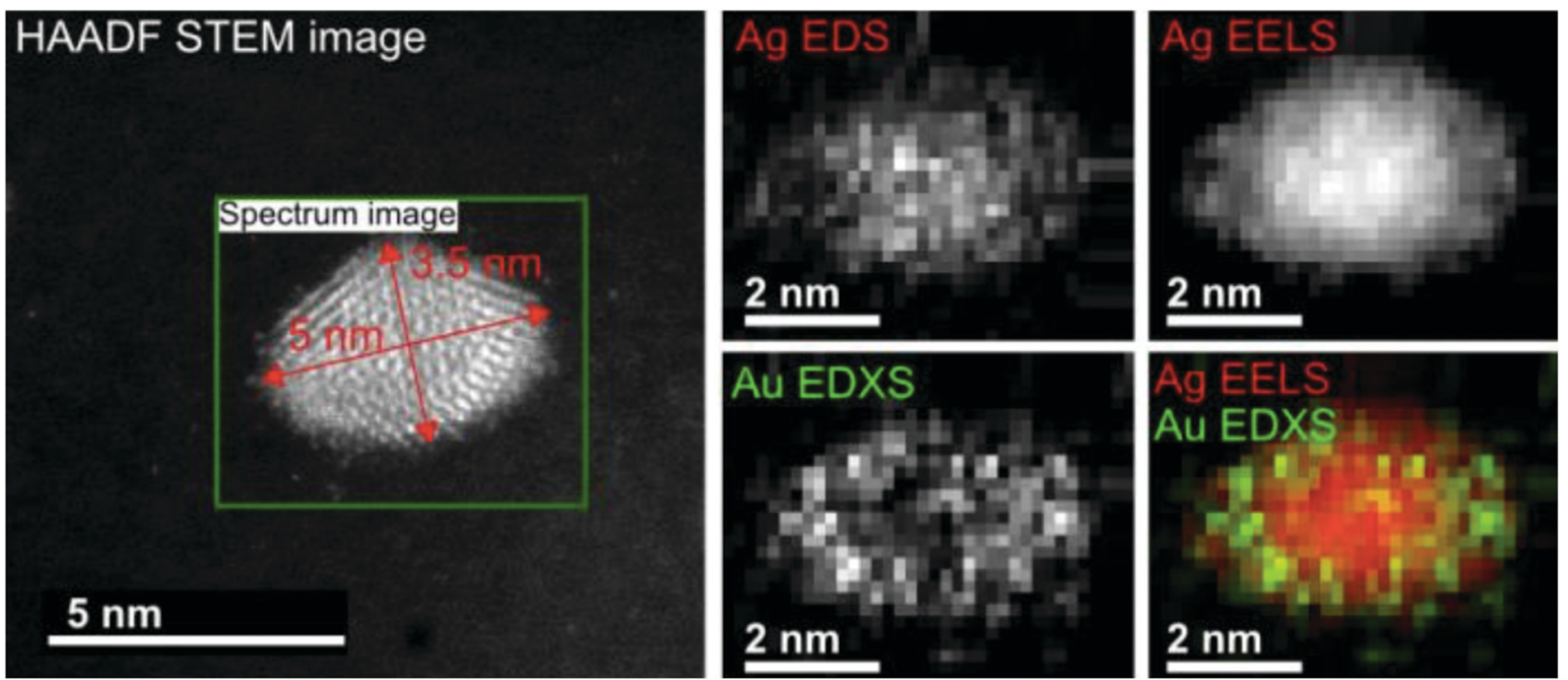

Fig. 13 EELS and EDXS elemental maps of an Ag@Au core@ shell nanocluster acquired at a tilt angle of $65^{\circ}$. The boxed region in the HAADF STEM image indicates the area of the mapped region and the size of the cluster. The maps have been generated from the EDXS signal of the Ag and Au L-lines, and for the EELS signal the Ag M-edge intensity was extracted. The color overlay was formed from the Ag EELS and the Au EDXS signal. Reprinted from ref. 78 under the Creative Common License.

the observed intermetallic diffusion was theoretically studied employing density functional theory. It could be shown that the presence of oxygen lowers the diffusion barrier for the migration of Ni core atoms through the Au shell to the surface. ${ }^{109}$

A recent study of iron triade nanoparticles passivated by gold layers was conducted to probe for the temperature dependent alloying in an oxygen-free vacuum. ${ }^{111}$ Starting from the synthesis of spherical Fe@Au, Co@Au, and Ni@Au core@shell clusters with centralized cores, the same heating procedures were applied to all combinations inside the electron microscope. The three different combinations exhibit rather different responses to thermal treatment: at $400{ }^{\circ} \mathrm{C}$, the Fe core breaks up into several smaller decentralized cores still surrounded by $\mathrm{Au}$ atoms and a complete alloying is only observed at $1100{ }^{\circ} \mathrm{C}$ after entering the liquid phase. For Co@Au, a structural transition to a decentralized Co core is observed at $400{ }^{\circ} \mathrm{C}$ (see Fig. 14) which is stable up to the melting temperature of $1100{ }^{\circ} \mathrm{C}$ when complete alloying takes place. In contrast, the Ni@Au clusters undergo a complete mixing already at $400{ }^{\circ} \mathrm{C}$, i.e. alloying takes place by diffusion through lattice before any liquid phase would set in (Fig. 15).

Initially, the three bimetallic cluster combinations were not expected to exhibit such different behaviour. Searching for an explanation, theoretical calculations were performed employing two different approaches, one based on the embedded atom method (EAM) and the other using a density functional theory (DFT) ansatz. ${ }^{111} \mathrm{~A}$ comparison of vacancy energies at the EAM and the DFT level, calculated for the four metals, and studies of Helmholtz free energies of the three bimetallic systems at various degrees of intermixing, provides a satisfactory explanation.

The size and shape dependent plasmonic properties of metal clusters promise interesting applications in the areas of photonics, optical sensing, or surface enhanced Raman spectroscopy (SERS).
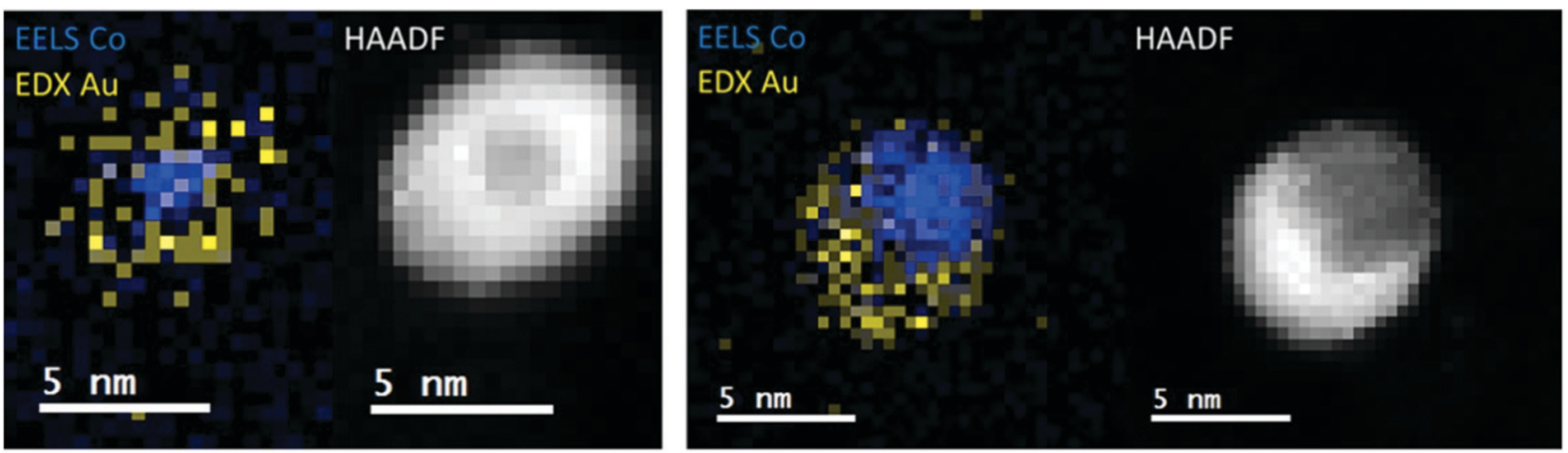

Fig. 14 EELS Co observations and EDX Au observations as well as complementary HAADF scans of temperature-induced structural transition from centralized (left) to decentralized (right) Co-Au clusters. The temperatures correspond to $25^{\circ} \mathrm{C}$ (left) and $400{ }^{\circ} \mathrm{C}$ (right). Reprinted from ref. 111 with the permission of the American Chemical Society. 

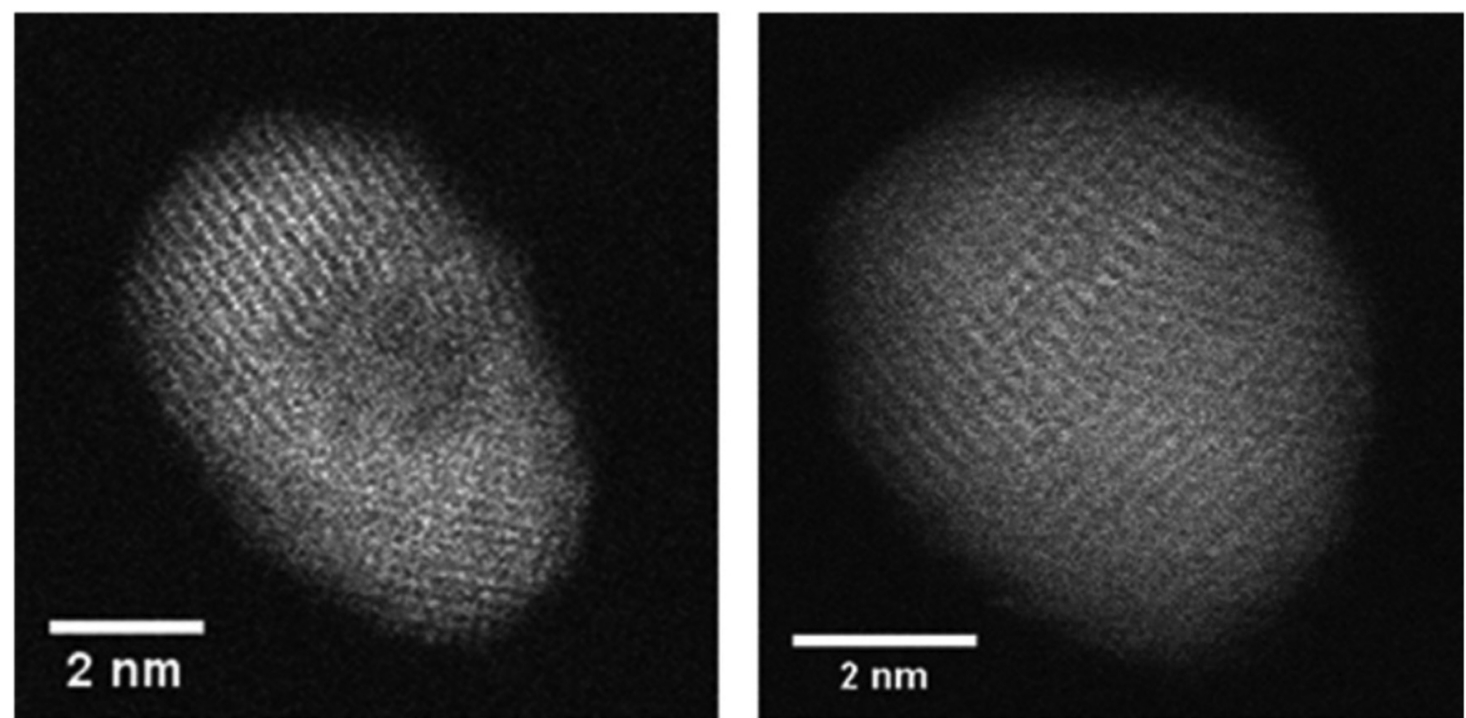

Fig. 15 HAADF observation of temperature-induced rearrangements within a Ni-Au cluster. The cluster is embedded centrally inside an Au shell. At $300-400{ }^{\circ} \mathrm{C}$ the $\mathrm{Ni}$ and $\mathrm{Au}$ atoms start to form an alloy, still exhibiting a lattice structure. Reprinted from ref. 111 with the permission of the American Chemical Society.

As shown in ref. 63, the optical absorption of a deposited cluster sample of Ag@Au core@shell clusters can be shifted between the silver cluster resonance around $450 \mathrm{~nm}$ and the gold cluster absorption near $550 \mathrm{~nm}$ depending on the number ratio of $\mathrm{Ag}$ atoms in the core and $\mathrm{Au}$ atoms in the shell. The study also demonstrated the use of such core@shell cluster samples for SERS by measuring Raman spectra of 4-methylbenzenethiol (4-MBT) enhanced by clusters of different core and shell contents in combination with different laser excitation wavelengths. ${ }^{63}$ As expected, blue laser light excitation yielded the best Raman intensities in combination with pure silver clusters. For red light, a 1:1 ration of $\mathrm{Ag}$ and $\mathrm{Au}$ contents yielded the best Raman enhancement.

While the absorption spectroscopy in ref. 63 required a sample of deposited nanoparticles that consisted of some cluster size distribution always present in our synthesis, the plasmon resonance of a single particle can be probed by electron energy loss spectroscopy (EELS). Fig. 16 shows the example of an elongated Ag@Au core@shell particle or nanorod that was created by doping a helium droplet beam at nozzle temperature of $6.7 \mathrm{~K}$ and 20 bar pressure. ${ }^{75}$ As mentioned before, by choosing the helium source conditions and the amount of doping, the synthesized particle shape and distribution can be controlled to some extent. As seen in panels (a) to (d), the nanorod has a regular shape with a connected silver core and an absorption peaked near $1.95 \mathrm{eV}$, i.e. about $640 \mathrm{~nm}$. The plasmon resonance map shows the spatial distribution with the maximum of the absorption lying near the ends of the particle. ${ }^{75}$

\subsection{Metal oxide nanoparticles}

Like their respective bulk versions, metal oxide nanoparticles are expected to become efficient nano catalysts for reactions like heterogeneous oxidation in depollution, photocatalysis, or hydrogen production with the advantage of their large surface to volume ratio. Only recently, we began to explore the potential of helium droplet assisted synthesis of metal oxide nanoparticles for the fundamental investigation of the morphology, stability, and electronic properties of these particles.

5.3.1 $\quad \mathbf{V}_{2} \mathbf{O}_{5}$. Transition metal oxides have unique properties originating from the characteristics of the outer d-band electrons. Vanadium oxide is a prominent representative of this group of materials, and clusters consisting of it have been object of active research due to their numerous oxidation states and the relatively high abundance of the material. Among the vanadium oxides, vanadium pentoxide is used in various industrial processes as catalyst, e.g. in the oxidation of ethanol to ethanal and in the production of phthalic anhydride, polyamide, oxalic acid and further products, or in the selective catalytic reduction of $\mathrm{NO}_{x}$ with $\mathrm{NH}_{3}$ in automobile exhaust systems.

In order to produce $\mathrm{V}_{2} \mathrm{O}_{5}$ nanoparticles and deposit them on solid substrates, we heated $\mathrm{V}_{2} \mathrm{O}_{5}$ powder with a purity of $99.9 \%$, kept in a small quartz crucible (Kurt Lesker EVC2Q), to temperatures between 1000 and $1200 \mathrm{~K}$, which yielded a vapour pressure of about $10^{-3}$ mbar. $^{69}$ Helium droplets were generated at 20 bar stagnation pressure and various nozzle temperatures for different droplet size distributions. The TOF-MS served as diagnostic tool for the observation of aggregation products formed in the droplets. To our surprise, no measurable number of $\mathrm{V}_{2} \mathrm{O}_{5}$ molecules showed up in the mass spectra, but significant abundances of even multiples of $\mathrm{V}_{2} \mathrm{O}_{5}$ such as $\left(\mathrm{V}_{2} \mathrm{O}_{5}\right)_{2}$ and $\left(\mathrm{V}_{2} \mathrm{O}_{5}\right)_{4}$. The experimental measurements were accompanied by a computational study on a single layer of bulk vanadium(v) oxide, which suggests a preferred sublimation of the dimers $\left(\mathrm{V}_{2} \mathrm{O}_{5}\right)_{2}{ }^{69}$

For the deposition of $\mathrm{V}_{2} \mathrm{O}_{5}$ nanoparticles, ${ }^{128}$ the nozzle temperature was lowered to 5.4, 6.7 and $8 \mathrm{~K}$, which corresponds to average sizes of $1.68 \times 10^{10}, 6 \times 10^{7}$ and $5.31 \times 10^{6}$ helium atoms per droplet, respectively. Larger $\mathrm{V}_{2} \mathrm{O}_{5}$ nanoparticles of 

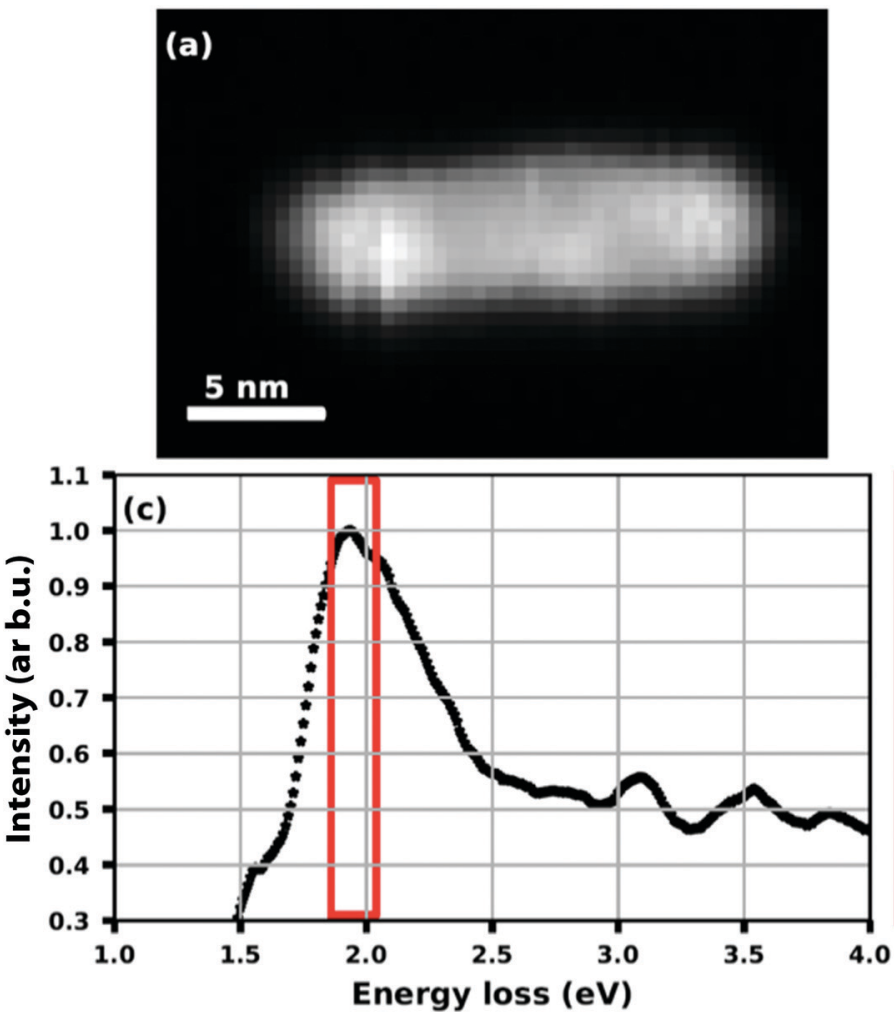
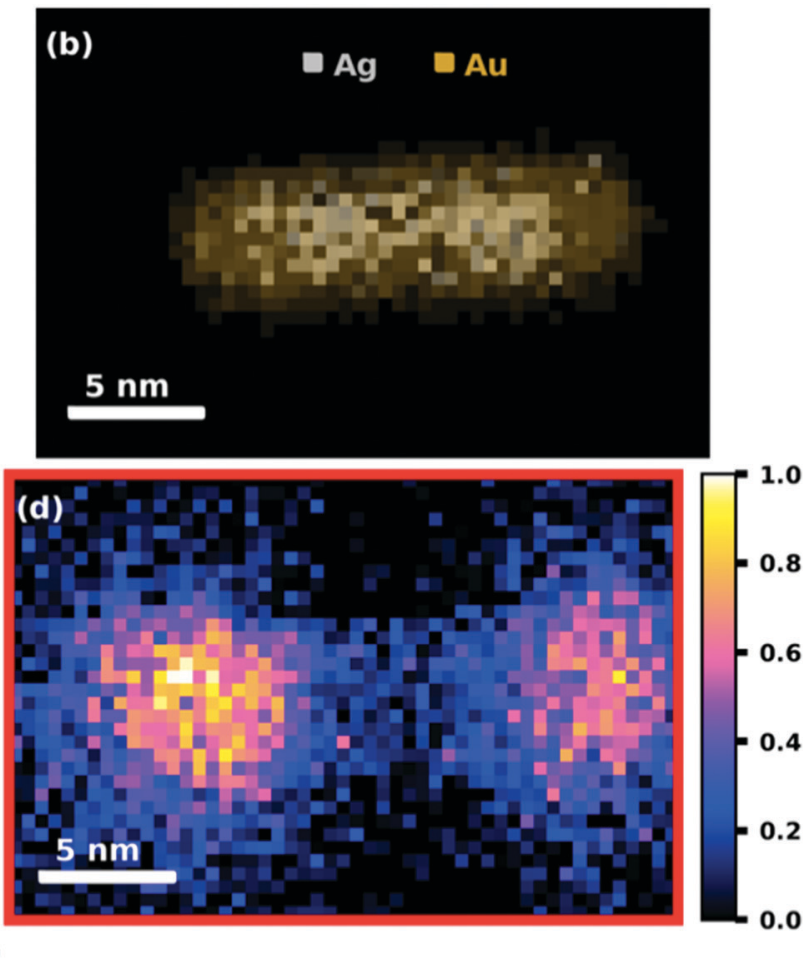

Fig. 16 HAADF image (a) of a Ag@Au coreashell nanorod with (b) an elemental map extracted from the EDS data showing the Ag core and Au shell structure. (c) A plot of the processed EELS data integrated over the whole image area where the red framed section is used for (d) a plasmon resonance map at $(1.95+0.09)$ eV. Reproduced from ref. 75 [A. Schiffmann, D. Knez, F. Lackner, M. Lasserus, R. Messner, M. Schnedlitz, G. Kothleitner, F. Hofer and W. E. Ernst, J. Appl. Phys., 2019, 125, 023104], with the permission of AIP Publishing.

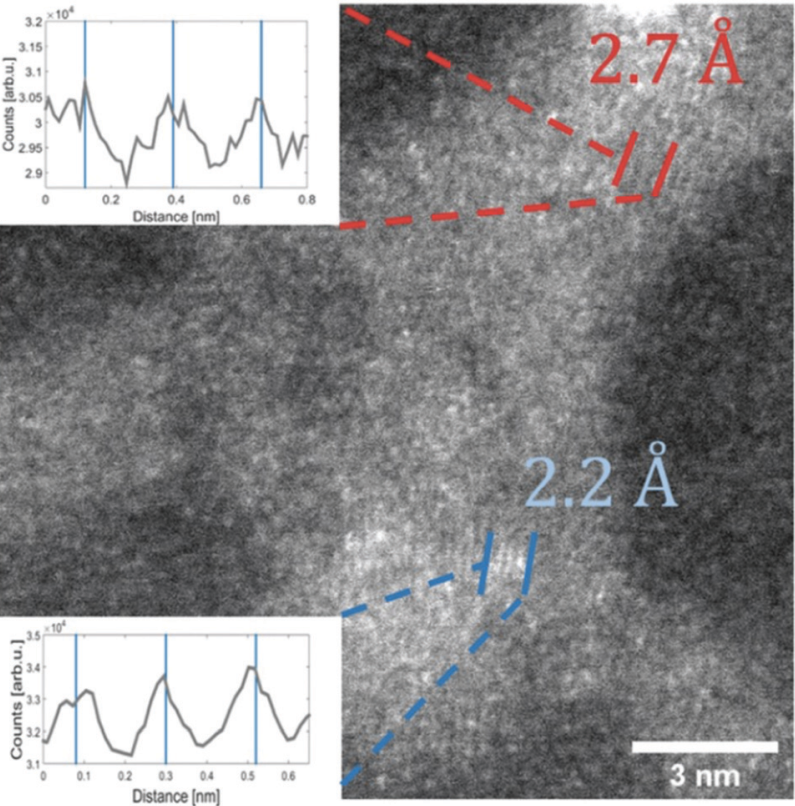

Fig. 17 STEM HAADF image of a $\mathrm{V}_{2} \mathrm{O}_{5}$ particle (synthesised at $5.4 \mathrm{~K}$ cold head temperature). Two regions are marked where lattice constants could be extracted from the image. The respective intensity profile between the lines is shown in the inset. The black lines indicate a group of planes with a spacing of $2.7 \AA$ the blue lines mark a group of planes with a spacing of $2.2 \AA$. Ref. 128 - reprinted with permission of the PCCP Owner Societies.
$10 \mathrm{~nm}$ side length were deposited this way as depicted in Fig. 17. Lattice constants were extracted from a large number of electron microscope images with the drawback that only projections of the deposited particles on the substrate are obtained. However, the large number of analyzed particles and comparison with tabulated lattice structures for vanadium oxides in the literature, supported by the observed UV-visible absorption spectra of our samples (Fig. 18), let us conclude that the $\mathrm{V}_{2} \mathrm{O}_{5}$ nanoparticles synthesized in helium droplets stayed intact during the deposition.

5.3.2 ZnO. Zinc oxide is a semiconductor that is used, for example, in radiation detectors for measuring electromagnetic radiation or in photocatalysts for breaking down organic pollutants. A recent monograph in the Nanoscience \& Nanotechnology Series of the Royal Society of Chemistry is devoted to 'ZnO Nanostructures' with the emphasis on 'Fabrication and Applications. ${ }^{, 129}$ We have approached the synthesis of $\mathrm{ZnO}$ nanoparticles by creating $\mathrm{Zn}$ clusters in helium droplets with controlled oxidation after the deposition. In order to combine the catalytic properties of $\mathrm{ZnO}$ with a plasmonic enhancement by a silver core for efficient photocatalysis, we synthesized Ag@ZnO core@shell particles. In this case, the helium droplet assisted creation of Ag@Zn particle was the starting point followed by oxidation that was carefully checked by scanning transmission electron microscopy (STEM). ${ }^{130}$ Using different droplet beam conditions, smaller spherical core@shell clusters and larger extended structures were deposited on the same 


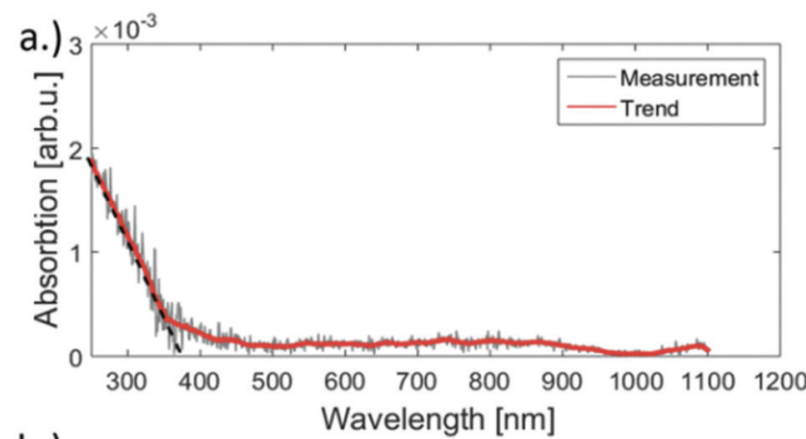

b.)

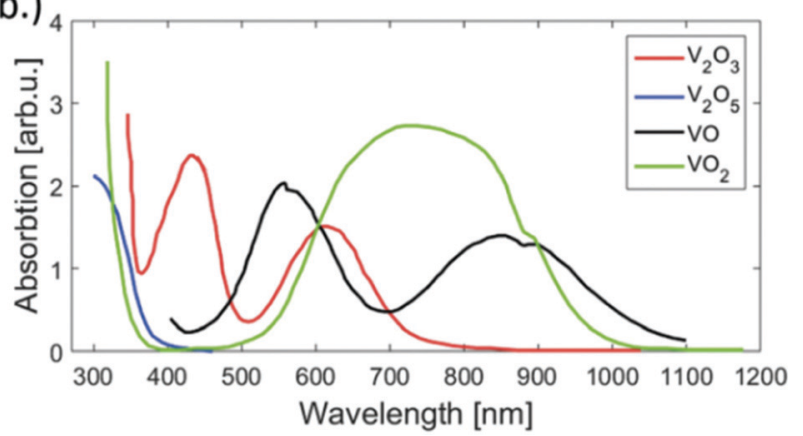

Fig. 18 UV-vis absorption spectra of the synthesised $\mathrm{V}_{2} \mathrm{O}_{5}$ clusters on fused silica (a) and reference spectra taken from ref. 141 (b). Apparently our sample has significant absorption only below $450 \mathrm{~nm}$ but no bands above. The spectrum was acquired by subtracting the signal of an uncovered plane spot on the substrate from the spot of cluster deposition. When comparing the reference spectra of various vanadium oxides to the measurement, only $\mathrm{V}_{2} \mathrm{O}_{5}$ has no absorption in the range from 450 to $1100 \mathrm{~nm}$. Ref. 128 - reprinted with permission of the PCCP Owner Societies.

substrate exposed to oxygen and imaged as shown in Fig. 19a. The picture shows a nice uniform $\mathrm{ZnO}$ shell around the brighter silver core. Fig. 19b provides a high resolution insight into the $\mathrm{ZnO}$ structure that was identified as $2 \mathrm{D}$ projection of the hexagonal unit cell of wurtzite $\mathrm{ZnO}$ in [001] direction. Ultraviolet photoelectron spectroscopy (UPS) indicates a fully oxidized shell layer for the particles studied by STEM. The plasmonic properties of the produced spherical Ag@ZnO core@shell particles were investigated by two-photon photoelectron (2PPE) spectroscopy. Upon excitation of the localized surface plasmon resonance in $\mathrm{Ag}$ at around $3 \mathrm{eV}$, plasmonic enhancement leads to the liberation of electrons with high kinetic energy. ${ }^{130}$

5.3.3 CoO. Cobalt oxides represent another example of a catalytically active material for the oxidation of ammonia to nitric acid. As nanoparticles, cobalt(II,III) oxide is used as an electrode in some lithium-ion batteries. Similar to the creation of $\mathrm{ZnO}$ particles, Co clusters were assembled in helium droplets, deposited, and controlled oxidized. ${ }^{131}$ Energy dispersive X-ray spectroscopy confirmed the elemental composition, and evaluation of high resolution TEM images are in agreement with the lattice distances of the $\mathrm{CoO}(111)$ and (200) planes. Samples with about $25 \%$ surface coverage were then investigated in the XUV energy range between 50 and $70 \mathrm{eV}$ generated by high harmonic generation (HHG) of the output of a $785 \mathrm{~nm}, 3.3 \mathrm{~mJ}, 3 \mathrm{kHz}$, 40 fs full-width-at-half-maximum (FWHM) Ti:sapphire laser
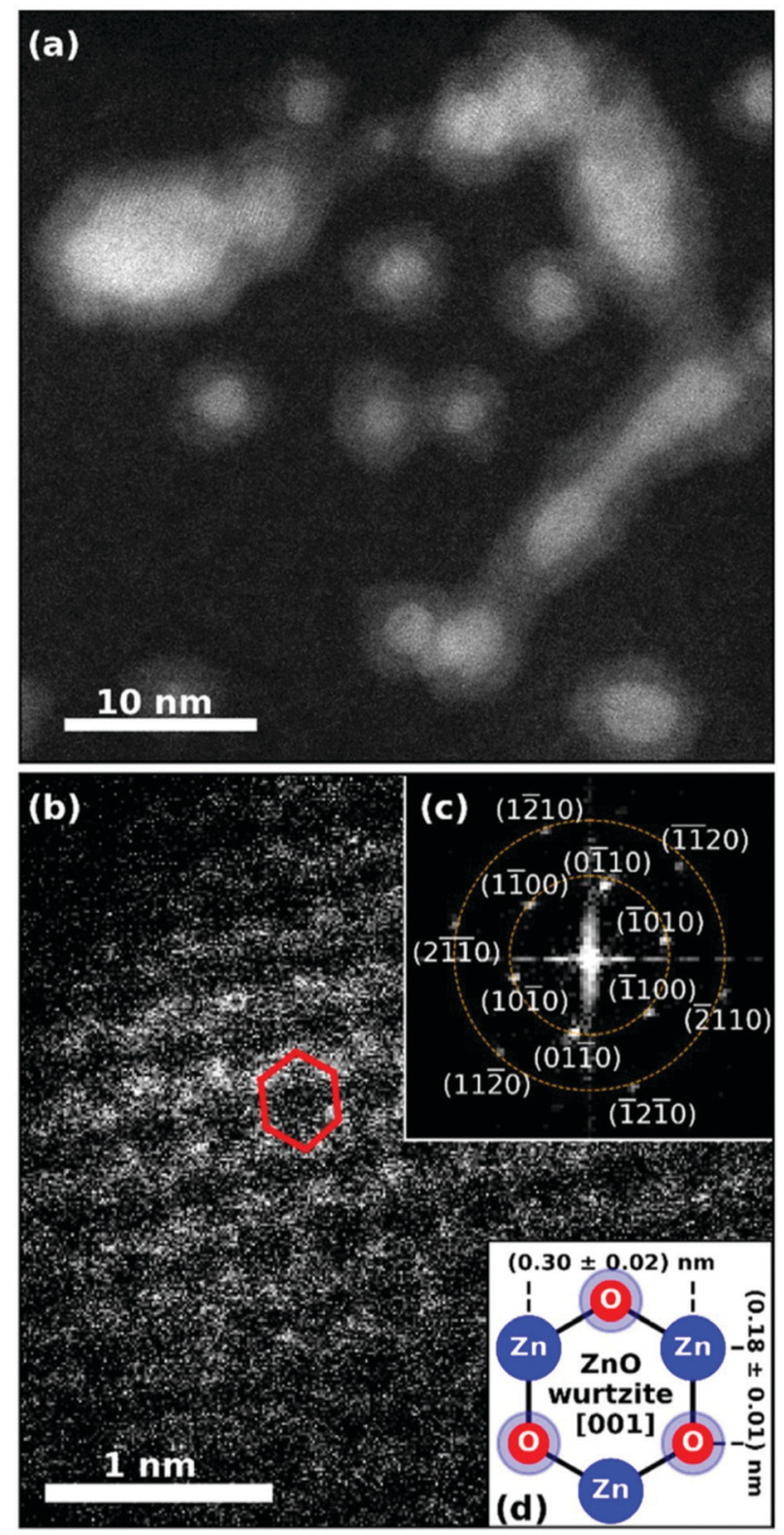

Fig. 19 (a) STEM HAADF image of the synthesized AgaZnO nanosized particles. Spherically shaped as well as wire-like structures can be identified. The coreashell structure is obvious as silver cores appear brighter than zinc oxide shells. The smaller spherical particles and the elongated larger particles were created at different helium droplet beam conditions and were deposited on the same substrate subsequently. (b) Detailed HAADF image showing $\mathrm{ZnO}$ along the [001] axis with the hexagonal structure highlighted in red for a selected unit cell. Inset plot (c) shows the fast Fourier transform (FFT) of the STEM image, yielding periodicities of $(0.30+0.02) \mathrm{nm}$ and $(0.18+0.01) \mathrm{nm}$ marked by the dashed orange circles. The sixfold symmetry of the FFT pattern is identified and all visible features are assigned. The schematic in inset plot (d) explains the origin of the two observed lengths within the $2 \mathrm{D}$ projection of the hexagonal unit cell of wurtzite $\mathrm{ZnO}$ in [001] direction. Reprinted from ref. 130 under the Creative Common License.

system. The work ${ }^{131}$ demonstrated the feasibility of studying nanoparticle samples prepared in helium droplets with tabletop ultrafast laser sources. 


\section{Summary and outlook}

With this perspective, we have shown that helium droplets provide an excellent cryogenic environment for building clusters in a controlled composition atom by atom without any additional chemicals or additives. By varying the helium droplet source conditions and the vapour pressure of the dopants, size, shape, and elemental mixture can be influenced within certain distributions. While the method will not be suitable for mass production of cluster material, it is an excellent means for fundamental studies on clean nanoparticles without any contamination answering questions about nanoscale elemental mixing and alloying or phase transitions in general. As shown in our work depending on the particle size, surface coverages in the range between $10 \%$ and a monolayer can be achieved in realistic operating times.

As diagnostics of the generated particles inside the helium droplets, basically any technique known from molecular beam spectroscopy can be applied, such as laser spectroscopy, ionization with mass analysis, or double resonance. ${ }^{35,71}$ The analytics can be continued within the beam apparatus even after the soft landing deposition by employing a quartz crystal microbalance that will measure the effect of either adding reactants or of depleting the coverage by resonant laser heating. Once deposited on exchangeable solid substrates that may be mounted on a rotatable holder, the created samples are transported under UHV to various surface diagnostic instruments.

Single particle diagnostics are provided by a large range of electron microscopy tools such as transmission electron microscopes or scanning electron microscopes equipped with highangular annular dark-field technique (HAADF), energy-dispersive X-ray spectroscopy (EDXS), and the electron energy loss spectroscopy (EELS). At the loss of individual particle resolution, we have investigated samples with UV-optical absorption spectroscopy and photoelectron spectroscopy. A recently set up femtosecond laser lab $^{46}$ in combination with high harmonic generation ${ }^{131}$ and the new NanoESCA lab will open up new opportunities like timeresolved photoemission electron microscopy, ${ }^{132}$ ultrafast charge carrier dynamics measurements, ${ }^{133}$ or time-resolved magnetic circular dichroism detection. ${ }^{134}$ For polar particles in helium droplets, Merthe and Kresin demonstrated electrostatic deflection as direct measure in the doped helium droplet beam, ${ }^{135}$ and built, with the group of Schäfer in Darmstadt, a setup for a magnetic deflection experiment. ${ }^{136}$ In this way, the magnetic properties of synthesized nanoparticles could directly be probed.

On the theoretical side, He density functional theory has been mentioned as the currently most suitable simulation method, allowing for a quantum treatment of $\mathrm{He}$ droplets containing vortices as well as impurities, but also having its limits with respect to droplet size and the ability to describe large collective excitations. Collision time estimates for impurities, derived from a simplified model, have been linked to growth scenarios of metallic structures inside He droplets. The deposition process could be identified as minimally perturbative to the grown structures. Suitable theoretical treatments for He-grown metallic clusters depend on their actual size, ranging from first principle treatments for smallest systems to force field descriptions based on the embedded atom model for particles in the nanometer range. A theoretical treatment of diffusion processes for even larger metallic structures via cellular automata on a coarse-grained lattice has been demonstrated. Challenges for future developments, in particular with respect to surface reactions on catalytically active sites of the synthesized mixed-metallic structures, will be the suitable coupling of various levels of computational treatment, ${ }^{137}$ and the implementation of novel machine learning techniques. ${ }^{117,118}$

Especially core@shell cluster configurations for catalysis or photocatalysis seem to be very promising in this context, ${ }^{138}$ as has been proven by recent experiments. ${ }^{139,140}$ We think that a combination of a theoretical screening via systematic first principle studies with the subsequent, He-assisted creation of the most promising core@shell candidates, followed by their deposition on suitable substrates, could accelerate the ongoing search for optimum configurations. A test apparatus for small scale catalysis experiments is in preparation.

\section{Conflicts of interest}

There are no conflicts to declare.

\section{Acknowledgements}

This research has been supported by the Austrian Science Fund (FWF) under Grant No. P 29893-N36 and by the FWF in cooperation with the Christian Doppler Research Association (CDG) under Grant No. PIR8-N34. Further support by NAWI Graz is gratefully acknowledged. The described cluster lab was built in 2011-2014, supported by the European Commission and the Styrian Government within the ERDF program (7th framework). An infrastructure grant from the HRSM program of the Austrian Government enabled the aquisition of the NanoESCA instrument. The authors are grateful to F. Lackner, A. Schiffmann, T. Jauk, M. Schnedlitz, M. Lasserus, D. Knez, H. Fitzek, G. Kothleitner, F. Hofer and M. P. de Lara-Castells for the highly productive years of joint collaboration.

\section{References}

1 M. Moskovits and J. E. Hulse, J. Chem. Soc., Faraday Trans. 2, 1977, 73, 471-484.

2 M. D. Morse, Chem. Rev., 1986, 86, 1049-1109.

3 H. Haberland, Springer Series in Chemical Physics, Clusters of Atoms and Molecules: Theory, Experiment, and Clusters of Atoms, Springer, Berlin, 1994.

4 W. A. de Heer, Rev. Mod. Phys., 1993, 65, 611-676.

5 U. Kreibig and M. Vollmer, Optical Properties of Metal Clusters, Springer, Berlin, 1995, p. 535.

6 W. A. de Heer and P. Milani, Rev. Sci. Instrum., 1991, 62, 670-677.

7 I. M. Billas, A. Châtelain and W. A. de Heer, Science, 1994, 265, 1682-1684. 
8 R. Moro, X. Xu, S. Yin and W. A. de Heer, Science, 2003, 300, 1265-1269.

9 E. Roduner, Chem. Soc. Rev., 2006, 35, 583-592.

10 J. Bansmann, S. Baker, C. Binns, J. Blackman, J.-P. Bucher, J. Dorantes-Dávila, V. Dupuis, L. Favre, D. Kechrakos, A. Kleibert, K.-H. Meiwes-Broer, G. Pastor, A. Perez, O. Toulemonde, K. Trohidou, J. Tuaillon and Y. Xie, Surf. Sci. Rep., 2005, 56, 189-275.

11 M. Martins and W. Wurth, J. Phys.: Condens. Matter, 2016, 28, 503002.

12 G. Schmid and L. F. Chi, Adv. Mater., 1998, 10, 515-526.

13 W. Schöllkopf and J. P. Toennies, Science, 1994, 266, 1345-1348.

14 E. W. Becker, R. Klingelhöfer and P. Lohse, Zeitschrift für Naturforschung A, 1962, 17, 432-438.

15 A. Scheidemann, J. P. Toennies and J. A. Northby, Phys. Rev. Lett., 1990, 64, 1899-1902.

16 S. Goyal, D. L. Schutt and G. Scoles, J. Phys. Chem., 1993, 97, 2236-2245.

17 M. Hartmann, R. E. Miller, J. P. Toennies and A. Vilesov, Phys. Rev. Lett., 1995, 75, 1566-1569.

18 G. Auböck, J. Nagl, C. Callegari and W. E. Ernst, J. Phys. Chem. A, 2007, 111, 7404-7410.

19 S. Grebenev, J. P. Toennies and A. F. Vilesov, Science, 1998, 279, 2083-2086.

20 M. Hartmann, F. Mielke, J. P. Toennies, A. F. Vilesov and G. Benedek, Phys. Rev. Lett., 1996, 76, 4560-4563.

21 L. F. Gomez, K. R. Ferguson, J. P. Cryan, C. Bacellar, R. M. P. Tanyag, C. Jones, S. Schorb, D. Anielski, A. Belkacem, C. Bernando, R. Boll, J. Bozek, S. Carron, G. Chen, T. Delmas, L. Englert, S. W. Epp, B. Erk, L. Foucar, R. Hartmann, A. Hexemer, M. Huth, J. Kwok, S. R. Leone, J. H. S. Ma, F. R. N. C. Maia, E. Malmerberg, S. Marchesini, D. M. Neumark, B. Poon, J. Prell, D. Rolles, B. Rudek, A. Rudenko, M. Seifrid, K. R. Siefermann, F. P. Sturm, M. Swiggers, J. Ullrich, F. Weise, P. Zwart, C. Bostedt, O. Gessner and A. F. Vilesov, Science, 2014, 345, 906-909.

22 J. Higgins, C. Callegari, J. Reho, F. Stienkemeier, W. E. Ernst, M. Gutowski and G. Scoles, J. Phys. Chem. A, 1998, 102, 4952-4965.

23 J. Higgins, W. E. Ernst, C. Callegari, J. Reho, K. K. Lehmann, G. Scoles and M. Gutowski, Phys. Rev. Lett., 1996, 77, 4532-4535.

24 K. Nauta and R. E. Miller, Science, 1999, 283, 1895-1897. 25 K. Nauta and R. E. Miller, Science, 2000, 287, 293-295.

26 J. Higgins, C. Callegari, J. Reho, F. Stienkemeier, W. E. Ernst, K. K. Lehmann, M. Gutowski and G. Scoles, Science, 1996, 273, 629-631.

27 J. Nagl, G. Auböck, A. W. Hauser, O. Allard, C. Callegari and W. E. Ernst, Phys. Rev. Lett., 2008, 100, 063001.

28 A. W. Hauser and W. E. Ernst, Phys. Chem. Chem. Phys., 2011, 13, 18762-18768.

29 C. Giese, F. Stienkemeier, M. Mudrich, A. W. Hauser and W. E. Ernst, Phys. Chem. Chem. Phys., 2011, 13, 18769-18780.

30 C. Stark and V. V. Kresin, Phys. Rev. B: Condens. Matter Mater. Phys., 2010, 81, 085401.
31 M. Theisen, F. Lackner and W. E. Ernst, J. Phys. Chem. A, 2011, 115, 7005-7009.

32 O. Bünermann and F. Stienkemeier, Eur. Phys. J. D, 2011, 61, 645-655.

33 M. Theisen, F. Lackner and W. E. Ernst, Phys. Chem. Chem. Phys., 2010, 12, 14861-14863.

34 J. P. Toennies and A. F. Vilesov, Angew. Chem., Int. Ed., 2004, 43, 2622-2648.

35 C. Callegari and W. E. Ernst, Helium Droplets as Nanocryostats for Molecular Spectroscopy-from the Vacuum Ultraviolet to the Microwave Regime, American Cancer Society, 2011.

36 F. Stienkemeier, F. Meier and H. O. Lutz, J. Chem. Phys., 1997, 107, 10816-10818.

37 E. Loginov and M. Drabbels, J. Chem. Phys., 2012, 136, 154302.

38 L. Kazak, S. Göde, K.-H. Meiwes-Broer and J. Tiggesbäumker, J. Phys. Chem. A, 2019, 123, 5951-5956.

39 M. Mudrich, B. Forkl, S. Müller, M. Dvorak, O. Bünermann and F. Stienkemeier, Rev. Sci. Instrum., 2007, 78, 103106.

40 R. Katzy, M. Singer, S. Izadnia, A. C. LaForge and F. Stienkemeier, Rev. Sci. Instrum., 2016, 87, 013105.

41 M. Ratschek, M. Koch and W. E. Ernst, J. Chem. Phys., 2012, 136, 104201.

42 F. Lindebner, A. Kautsch, M. Koch and W. E. Ernst, Int. J. Mass Spectrom., 2014, 365-366, 255-259.

43 M. Lasserus, M. Schnedlitz, D. Knez, R. Messner, A. Schiffmann, F. Lackner, A. W. Hauser, F. Hofer and W. E. Ernst, Nanoscale, 2018, 10, 2017-2024.

44 R. Messner, A. Schiffmann, J. V. Pototschnig, M. Lasserus, M. Schnedlitz, F. Lackner and W. E. Ernst, J. Chem. Phys., 2018, 149, 024305.

45 M. Schnedlitz, D. Knez, M. Lasserus, F. Hofer, R. FernándezPerea, A. W. Hauser, M. Pilar de Lara-Castells and W. E. Ernst, J. Phys. Chem. C, 2020, 124, 16680-16688.

46 B. Thaler, S. Ranftl, P. Heim, S. Cesnik, L. Treiber, R. Meyer, A. W. Hauser, W. E. Ernst and M. Koch, Nat. Commun., 2018, 9, 4006.

47 R. E. Grisenti and J. P. Toennies, Phys. Rev. Lett., 2003, 90, 234501. 48 L. F. Gomez, E. Loginov, R. Sliter and A. F. Vilesov, J. Chem. Phys., 2011, 135, 154201.

49 A. Volk, P. Thaler, D. Knez, A. W. Hauser, J. Steurer, W. Grogger, F. Hofer and W. E. Ernst, Phys. Chem. Chem. Phys., 2016, 18, 1451-1459.

50 V. Mozhayskiy, M. N. Slipchenko, V. K. Adamchuk and A. F. Vilesov, J. Chem. Phys., 2007, 127, 094701.

51 E. Loginov, L. F. Gomez and A. F. Vilesov, J. Phys. Chem. A, 2011, 115, 7199-7204.

52 S. Yang, A. M. Ellis, D. Spence, C. Feng, A. Boatwright, E. Latimer and C. Binns, Nanoscale, 2013, 5, 11545-11553.

53 S. B. Emery, K. B. Rider, B. K. Little and C. M. Lindsay, J. Phys. Chem. C, 2013, 117, 2358-2368.

54 A. Volk, P. Thaler, M. Koch, E. Fisslthaler, W. Grogger and W. E. Ernst, J. Chem. Phys., 2013, 138, 214312.

55 P. Thaler, A. Volk, D. Knez, F. Lackner, G. Haberfehlner, J. Steurer, M. Schnedlitz and W. E. Ernst, J. Chem. Phys., 2015, 143, 134201. 
56 A. W. Hauser, A. Volk, P. Thaler and W. E. Ernst, Phys. Chem. Chem. Phys., 2015, 17, 10805-10812.

57 O. Gessner and A. F. Vilesov, Annu. Rev. Phys. Chem., 2019, 70, 173-198.

58 Y. A. Sergeev and C. F. Barenghi, J. Low Temp. Phys., 2009, 157, 429.

59 L. F. Gomez, E. Loginov and A. F. Vilesov, Phys. Rev. Lett., 2012, 108, 155302.

60 P. Thaler, A. Volk, F. Lackner, J. Steurer, D. Knez, W. Grogger, F. Hofer and W. E. Ernst, Phys. Rev. B: Condens. Matter Mater. Phys., 2014, 90, 155442.

61 D. Spence, E. Latimer, C. Feng, A. Boatwright, A. M. Ellis and S. Yang, Phys. Chem. Chem. Phys., 2014, 16, 6903-6906.

62 E. Gordon, A. Karabulin, V. Matyushenko, V. Sizov and I. Khodos, Phys. Chem. Chem. Phys., 2014, 16, 25229-25233.

63 F. Lackner, A. Schiffmann, M. Lasserus, R. Messner, M. Schnedlitz, H. Fitzek, P. Pölt, D. Knez, G. Kothleitner and W. E. Ernst, Eur. Phys. J. D, 2019, 73, 104.

64 M. Lasserus, D. Knez, M. Schnedlitz, A. Hauser, F. Hofer and W. E. Ernst, Nanoscale Adv., 2019, 1, 2276-2283.

65 W. D. Knight, K. Clemenger, W. A. de Heer, W. A. Saunders, M. Y. Chou and M. L. Cohen, Phys. Rev. Lett., 1984, 52, 2141-2143.

66 F. Baletto, C. Mottet and R. Ferrando, Phys. Rev. B: Condens. Matter Mater. Phys., 2001, 63, 155408.

67 P. Bartl, K. Tanzer, C. Mitterdorfer, S. Karolczak, E. Illenberger, S. Denifl and P. Scheier, Rapid Commun. Mass Spectrom., 2013, 27, 298-304.

68 S. Denifl, Eur. Phys. J.: Spec. Top., 2013, 222, 2017-2033.

69 M. Lasserus, M. Schnedlitz, R. Messner, F. Lackner, W. E. Ernst and A. W. Hauser, Chem. Sci., 2019, 10, 3473-3480.

70 J. Steurer, MSc thesis, Graz University of Technology, Graz, 2014.

71 M. Koch, J. Lanzersdorfer, C. Callegari, J. S. Muenter and W. E. Ernst, J. Phys. Chem. A, 2009, 113, 13347-13356.

72 T. Diederich, J. Tiggesbäumker and K.-H. Meiwes-Broer, J. Chem. Phys., 2002, 116, 3263-3269.

73 F. Xuan and C. Guet, Phys. Rev. A, 2017, 96, 043404.

74 E. Loginov, L. F. Gomez, N. Chiang, A. Halder, N. Guggemos, V. V. Kresin and A. F. Vilesov, Phys. Rev. Lett., 2011, 106, 233401.

75 A. Schiffmann, D. Knez, F. Lackner, M. Lasserus, R. Messner, M. Schnedlitz, G. Kothleitner, F. Hofer and W. E. Ernst, J. Appl. Phys., 2019, 125, 023104.

76 P. Thaler, A. Volk, M. Ratschek, M. Koch and W. E. Ernst, J. Chem. Phys., 2014, 140, 044326.

77 M. P. de Lara-Castells, N. F. Aguirre, H. Stoll, A. O. Mitrushchenkov, D. Mateo and M. Pi, J. Chem. Phys., 2015, 142, 131101.

78 G. Haberfehlner, P. Thaler, D. Knez, A. Volk, F. Hofer, W. E. Ernst and G. Kothleitner, Nat. Commun., 2015, 6, 8779.

79 M. Schnedlitz, MSc thesis, Graz University of Technology, Graz, 2017.

80 A. Schiffmann and H. Fitzek, unpublished.

81 C. Nacci and L. Grill, unpublished.

82 T. Jauk, MSc thesis, Graz University of Technology, Graz, 2019.
83 M. Barranco, R. Guardiola, S. Hernández, R. Mayol, J. Navarro and M. Pi, J. Low Temp. Phys., 2006, 142, 1.

84 F. Ancilotto, M. Barranco, F. Coppens, J. Eloranta, N. Halberstadt, A. Hernando, D. Mateo and M. Pi, Int. Rev. Phys. Chem., 2017, 36, 621-707.

85 M. Pi et al. ${ }^{4} \mathrm{He}-\mathrm{DFT}$ BCN-TLS: A Computer Package for Simulating Structural Properties and Dynamics of Doped Liquid Helium-4 Systems, 2016, https:/github.com/bcntls2016/.

86 R. J. Donnelly, Quantized Vortices in Helium II, Cambridge University Press, Cambridge, 1991.

87 F. Ancilotto, M. Pi and M. Barranco, Phys. Rev. B: Condens. Matter Mater. Phys., 2015, 91, 100503.

88 F. Coppens, F. Ancilotto, M. Barranco, N. Halberstadt and M. Pi, Phys. Chem. Chem. Phys., 2019, 21, 17423-17432.

89 F. Coppens, A. Leal, M. Barranco, N. Halberstadt and M. Pi, J. Low Temp. Phys., 2017, 187, 439-445.

90 F. Calvo, Phys. Rev. B, 2017, 95, 035429.

91 J. Höller, E. Krotscheck and R. E. Zillich, Eur. Phys. J. D, $2014,68,372$.

92 J. Höller, E. Krotscheck and R. E. Zillich, Eur. Phys. J. D, 2015, 69, 198.

93 J. Höller, E. Krotscheck and R. E. Zillich, Eur. Phys. J. D, 2015, 69, 198.

94 E. Krotscheck and R. E. Zillich, J. Chem. Phys., 2016, 145, 244317. 95 A. Hernando, M. Barranco, R. Mayol, M. Pi and F. Ancilotto, Phys. Rev. B: Condens. Matter Mater. Phys., 2008, 78, 184515.

96 M. Lasserus, PhD thesis, Graz University of Technology, 2020.

97 R. Fernández-Perea, L. F. Gómez, C. Cabrillo, M. Pi, A. O. Mitrushchenkov, A. F. Vilesov and M. P. de LaraCastells, J. Phys. Chem. C, 2017, 121, 22248-22257.

98 A. Volk, D. Knez, P. Thaler, A. W. Hauser, W. Grogger, F. Hofer and W. E. Ernst, Phys. Chem. Chem. Phys., 2015, 17, 24570-24575.

99 R. Thouy, N. Olivi-Tran and R. Jullien, Phys. Rev. B: Condens. Matter Mater. Phys., 1997, 56, 5321-5327.

100 A. W. Hauser, M. Schnedlitz and W. E. Ernst, Eur. Phys. J. D, 2017, 71, 150.

101 M. Schnedlitz, M. Lasserus, D. Knez, A. W. Hauser, F. Hofer and W. E. Ernst, Phys. Chem. Chem. Phys., 2017, 19, 9402-9408.

102 R. Ferrando, J. Jellinek and R. L. Johnston, Chem. Rev., 2008, 108, 845-910.

103 Nanoalloys - From Fundamentals to Emergent Applications, ed. F. Calvo, Elsevier, 2013.

104 Structure and Properties of Nanoalloys, ed. R. Ferrando, Elsevier, 2016.

105 J.-P. Palomares-Baez, E. Panizon and R. Ferrando, Nano Lett., 2017, 17, 5394-5401.

106 M. Jäger, R. Schäfer and R. L. Johnston, Adv. Phys.: X, 2018, 3, 1516514.

107 S. Liu, Z. Sun, Q. Liu, L. Wu, Y. Huang, T. Yao, J. Zhang, T. Hu, M. Ge, F. Hu, Z. Xie, G. Pan and S. Wei, ACS Nano, 2014, 8, 1886-1892.

108 V. Dupuis, A. Hillion, A. Robert, O. Loiselet, G. Khadra, P. Capiod, C. Albin, O. Boisron, D. Le Roy, L. Bardotti, F. Tournus and A. Tamion, J. Nanopart. Res., 2018, 20, 128. 
109 M. Schnedlitz, M. Lasserus, R. Meyer, D. Knez, F. Hofer, W. E. Ernst and A. W. Hauser, Chem. Mater., 2018, 30, 1113-1120.

110 D. Nelli and R. Ferrando, Nanoscale, 2019, 11, 13040-13050.

111 M. Schnedlitz, D. Knez, M. Lasserus, F. Hofer, R. FernándezPerea, A. W. Hauser, M. Pilar de Lara-Castells and W. E. Ernst, J. Phys. Chem. C, 2020, 124, 16680-16688.

112 S. M. Foiles, M. I. Baskes and M. S. Daw, Phys. Rev. B: Condens. Matter Mater. Phys., 1986, 33, 7983-7991.

113 Z. Qiao, H. Feng and J. Zhou, Phase Transitions, 2014, 87, 59-70.

114 X. W. Zhou, R. A. Johnson and H. N. G. Wadley, Phys. Rev. B: Condens. Matter Mater. Phys., 2004, 69, 10.

115 A. P. Sutton and J. Chen, Philos. Mag. Lett., 1990, 61, 139-146.

116 Y. Qi, T. Ç. Çă̆ı̆n, Y. Kimura and W. A. Goddard, Phys. Rev. B: Condens. Matter Mater. Phys., 1999, 59, 3527-3533.

117 Z. Claudio, R. Kevin, G. Aldo, F. Ádám, G. Nicola, B. Francesca and D. V. Alessandro, J. Chem. Phys., 2018, 148, 241739.

118 C. Zeni, K. Rossi, A. Glielmo and F. Baletto, Adv. Phys.: X, 2019, 4, 1654919.

119 M. Schnedlitz, R. Fernández-Perea, D. Knez, M. Lasserus, A. Schiffmann, F. Hofer, A. W. Hauser, M. P. de Lara-Castells and W. E. Ernst, J. Phys. Chem. C, 2019, 123, 20037-20043.

120 J. Ågren, Curr. Opin. Solid State Mater. Sci., 1996, 1, 355-360.

121 M. Cui, H. Lu, H. Jiang, Z. Cao and X. Meng, Sci. Rep., 2017, 7, 41990.

122 T. P. Senftle, S. Hong, M. M. Islam, S. B. Kylasa, Y. Zheng, Y. K. Shin, C. Junkermeier, R. Engel-Herbert, M. J. Janik, H. M. Aktulga, T. Verstraelen, A. Grama and A. C. T. van Duin, npj Comput. Mater., 2016, 2, 15011.

123 G. P. Bewley, D. P. Lathrop and K. R. Sreenivasan, Nature, 2006, 441, 588.

124 E. B. Gordon, R. Nishida, R. Nomura and Y. Okuda, JETP Lett., 2007, 85, 581-584.

125 P. Moroshkin, V. Lebedev, B. Grobety, C. Neururer, E. B. Gordon and A. Weis, EPL, 2010, 90, 34002.

126 E. B. Gordon, A. V. Karabulin, A. A. Morozov, V. I. Matyushenko, V. D. Sizov and I. I. Khodos, J. Phys. Chem. Lett., 2014, 5, 1072-1076.
127 E. B. Gordon, A. V. Karabulin, V. I. Matyushenko, V. D. Sizov and I. I. Khodos, Appl. Phys. Lett., 2012, 101, 052605.

128 M. Lasserus, D. Knez, F. Lackner, M. Schnedlitz, R. Messner, D. Schennach, G. Kothleitner, F. Hofer, A. W. Hauser and W. E. Ernst, Phys. Chem. Chem. Phys., 2019, 21, 21104-21108.

129 Y. Zhang, ZnO Nanostructures: Fabrication and Applications, Nanoscience \& Nanotechnology Series, The Royal Society of Chemistry, 2017, pp. 1-291.

130 A. Schiffmann, T. Jauk, D. Knez, H. Fitzek, F. Hofer, F. Lackner and W. E. Ernst, Nano Res., 2020, 13(11), 2979-2986.

131 A. Schiffmann, B. W. Toulson, D. Knez, R. Messner, M. Schnedlitz, M. Lasserus, F. Hofer, W. E. Ernst, O. Gessner and F. Lackner, J. Appl. Phys., 2020, 127, 184303.

132 K. Yu, D. Bayer, C. Wiemann, O. Gaier, M. Bauer and M. Aeschlimann, J. Nanomater., 2008, 2008, 249514.

133 M. E. Vaida, B. M. Marsh and S. R. Leone, Nano Lett., 2018, 18, 4107-4114.

134 F. Siegrist, J. A. Gessner, M. Ossiander, C. Denker, Y.P. Chang, M. C. Schröder, A. Guggenmos, Y. Cui, J. Walowski, U. Martens, J. K. Dewhurst, U. Kleineberg, M. Münzenberg, S. Sharma and M. Schultze, Nature, 2019, 571, 240-244.

135 D. J. Merthe and V. V. Kresin, J. Phys. Chem. Lett., 2016, 7, 4879-4883.

136 J. Liang, T. M. Fuchs, R. Schäfer and V. V. Kresin, Rev. Sci. Instrum., 2020, 91, 053202.

137 A. Bruix, J. T. Margraf, M. Andersen and K. Reuter, Nat. Catal., 2019, 2, 659-670.

138 Y. Hu, J. Huo, X. Wang and R. Wang, J. Phys.: Condens. Matter, 2017, 29, 435701.

139 V. A. Bharathan, R. Jain, C. S. Gopinath and C. P. Vinod, Catal. Sci. Technol., 2017, 7, 4489-4498.

140 P. B. O’Mara, P. Wilde, T. M. Benedetti, C. Andronescu, S. Cheong, J. J. Gooding, R. D. Tilley and W. Schuhmann, J. Am. Chem. Soc., 2019, 141, 14093-14097.

141 N. H. Choi, S.-k. Kwon and H. Kim, J. Electrochem. Soc., 2013, 160, A973-A979. 